\title{
Statistical atmospheric inversion of local gas emissions by coupling the tracer release technique and local-scale transport modelling: a test case with controlled methane emissions
}

\author{
Sébastien Ars ${ }^{1}$, Grégoire Broquet ${ }^{1}$, Camille Yver Kwok ${ }^{1}$, Yelva Roustan ${ }^{2}$, Lin Wu $^{1}$, Emmanuel Arzoumanian ${ }^{1}$, and \\ Philippe Bousquet ${ }^{1}$ \\ ${ }^{1}$ Laboratoire des sciences du climat et de l'environnement (LSCE/IPSL), CNRS-CEA-UVSQ, Université de Paris-Saclay, \\ Centre d'Etudes Orme des Merisiers, Gif-sur-Yvette, France \\ ${ }^{2}$ CEREA, Joint Laboratory École des Ponts ParisTech/EDF R\&D, Université Paris-Est, Champs-sur-Marne, France
}

Correspondence: Grégoire Broquet (gregoire.broquet@1sce.ipsl.fr)

Received: 20 October 2016 - Discussion started: 1 December 2016

Revised: 2 November 2017 - Accepted: 14 November 2017 - Published: 20 December 2017

\begin{abstract}
This study presents a new concept for estimating the pollutant emission rates of a site and its main facilities using a series of atmospheric measurements across the pollutant plumes. This concept combines the tracer release method, local-scale atmospheric transport modelling and a statistical atmospheric inversion approach. The conversion between the controlled emission and the measured atmospheric concentrations of the released tracer across the plume places valuable constraints on the atmospheric transport. This is used to optimise the configuration of the transport model parameters and the model uncertainty statistics in the inversion system. The emission rates of all sources are then inverted to optimise the match between the concentrations simulated with the transport model and the pollutants' measured atmospheric concentrations, accounting for the transport model uncertainty. In principle, by using atmospheric transport modelling, this concept does not strongly rely on the good colocation between the tracer and pollutant sources and can be used to monitor multiple sources within a single site, unlike the classical tracer release technique. The statistical inversion framework and the use of the tracer data for the configuration of the transport and inversion modelling systems should ensure that the transport modelling errors are correctly handled in the source estimation. The potential of this new concept is evaluated with a relatively simple practical implementation based on a Gaussian plume model and a series of inversions of controlled methane point sources using acetylene as a tracer gas. The experimental conditions are chosen so that they are suitable for the use of a Gaus-
\end{abstract}

sian plume model to simulate the atmospheric transport. In these experiments, different configurations of methane and acetylene point source locations are tested to assess the efficiency of the method in comparison to the classic tracer release technique in coping with the distances between the different methane and acetylene sources. The results from these controlled experiments demonstrate that, when the targeted and tracer gases are not well collocated, this new approach provides a better estimate of the emission rates than the tracer release technique. As an example, the relative error between the estimated and actual emission rates is reduced from $32 \%$ with the tracer release technique to $16 \%$ with the combined approach in the case of a tracer located $60 \mathrm{~m}$ upwind of a single methane source. Further studies and more complex implementations with more advanced transport models and more advanced optimisations of their configuration will be required to generalise the applicability of the approach and strengthen its robustness.

\section{Introduction}

Atmospheric pollution due to anthropogenic activities is a major issue both for air quality and for climate change. Industrial sites are known to emit a significant amount of pollutants and greenhouse gases. For instance in France, industrial emissions represent between 10 and $30 \%$ of major air pollutants, such as carbon and nitrous oxide (Bort and Langeron, 
2016). Currently, industries must list their emissions through national inventory reports, and some of them commit to reducing these emissions. However, the choice of an appropriate mitigation policy and the verification of its results require a good understanding of the emitting processes and a precise quantification of the emission rates. Industrial emissions are difficult to model and quantify because of the diversity and the temporal variability in the emitting processes.

Many emitting industrial sites have a typical size of $100 \mathrm{~m}^{2}-1 \mathrm{~km}^{2}$, and they emit pollutants from very specific locations within this area. Once emitted from a single or multiple point sources, the transport of these pollutants in the atmosphere over distances from 0.1 to several kilometres forms a distinct plume or multiple plumes, eventually merging at larger distance downstream. One approach developed to quantify the emissions from these sites involves atmospheric concentration measurements around the site, particularly across these emission plumes, and proxies of the atmospheric transport. These proxies are used to characterise the link between the emission rate and the structure and amplitude of the emission plume. The inversions of this link enable an estimate of the emission rates from the observed concentrations. Among the different techniques used to estimate emissions from concentrations is the tracer release method. It is often realised in the form of mobile continuous measurements across the emission plumes of the studied pollutant and of a tracer purposely emitted with a known rate as close as possible to the suspected pollutant source (Lamb et al., 1995). In this method, the proxy of the atmospheric transport is given by the relation between the tracer emission rate and the tracer concentrations. In practice, it provides estimates of the emissions of a site over a relatively short time window, i.e. typically a few hours during a given day, which generally corresponds to the time in which the tracer can be released or a mobile measurement can be conducted.

This approach is relatively simple to implement and enables instantaneous estimations for a large number of sites. Nevertheless, this technique encounters some limitations, particularly (i) when it is difficult to position the tracer emission close to the sources, (ii) when the sources are spread over a significant area compared with the distance between the sources and the location of the measured concentrations, or (iii) when targeting individual estimates of the different emission rates from multiple sources with plumes that overlap at a given site within distances at which the measurements can be conducted (Mønster et al., 2014; Roscioli et al., 2015). Typically, at industrial sites, pollutant sources may be sporadic and diffusive over a large area, their location can be difficult to reach and the spatial distribution of the emissions is not always precisely known, e.g. when considering transitory leakages or widespread and heterogeneous sources. In these cases, the tracer release method can induce errors in the flux estimation, since the tracer plume cannot be used by itself as an accurate proxy of the local transport from the targeted gas sources to the measurement locations. Moreover, this approach can hardly be used to provide an estimate of the different sources within a site.

Other techniques exploit atmospheric measurements using local atmospheric dispersion models to simulate the transport of the targeted gas from its sources to the measurement locations (Lushi and Stockie, 2010). Micrometeorological measurements are often conducted in parallel with those of the targeted gas concentrations in order to support the set-up of such models (Flesch et al., 2004, 2007; Gao et al., 2009). In theory, the model and the inversion of this proxy of the atmospheric transport can be applied for a point source or for a source with a known spread. In principle, they can also be applied to multiple sources. The principle of this technique is relatively simple, but the representation of the transport and emission spread by these models (even when they are constrained using micrometeorological measurements), and the separation of the different plumes associated with the different sources when targeting multiple sources can bear large uncertainties. In particular, the transport over short distances or timescales in a complex terrain can be characterised by complex turbulent structures which are difficult to match with a model, even when the underlying processes are taken into account. Moreover, when targeting several sources, this technique relies on the mathematical inversion of a square matrix characterising the atmospheric transport that links the set of sources to the observation data. This artificially requires extending or limiting the number of observation data from the measurement series to the number of sources to be quantified. It can lead to a loss of information or it can hide the fact that the problem is underconstrained when the plumes overlap too much.

The statistical inversion framework, which can be viewed as a generalised inversion technique, can account for uncertainties in the model. It can also address under- or overconstrained mathematical problems when constraining the source estimation with the correct number of observation data that corresponds to the complementary pieces of information in the measurements. In such a framework, a statistical estimate of the emission rates for the different targeted sources is derived to optimise the fit to the measurements, accounting for the statistical uncertainties in the source and transport modelling, in the measurements and in the prior knowledge of the source location and magnitude (Goyal et al., 2005). Statistical inversions using atmospheric transport models and atmospheric concentration measurements have been used for decades to infer surface sinks and/or sources of pollutants and greenhouse gases on continental to city scales (Gurney et al., 2003; Bré et al., 2015). However, the effectiveness of such approaches strongly relies on high accuracy of the transport modelling and on the ability to characterise statistics of the modelling uncertainties. It can also strongly rely on prior knowledge of emissions, in particular on the spatial distribution of the multiple sources within an industrial site for the type of applications considered in 
this study and on the ability to characterise the uncertainties with this knowledge.

This study describes a concept which combines the tracer release technique, local-scale transport modelling and the statistical inversion framework to improve the estimation of gas emissions from one or several point sources in an industrial site-scale configuration. It is based on the same measurement framework as the tracer release technique. It consists of using knowledge of the transport given by the tracercontrolled emission and concentration measurements to optimise the calibration of the transport model parameters and to assess the statistics of the model errors for the configuration of the inversion system. A practical implementation with a Gaussian plume model is demonstrated and its robustness is evaluated to illustrate the principle and the potential of the concept. This practical implementation is tested for the quantification of methane emissions during a time window of several hours using acetylene as a tracer gas and mobile measurements across the methane and acetylene plumes.

Methane is an important greenhouse gas with poorly known point source emissions (Saunois et al., 2016). Typical methane-emitting sites due to anthropogenic activities include waste-processing plants (wastewater treatment plants and landfills), oil and gas extraction and compressing sites and farms (Czepiel et al., 1996; Marik and Levin, 1996; Yver Kwok et al., 2015). These sites contain widespread and heterogeneous sources (like the basins in waste water treatment plants, the cells in landfills and the livestock in farms) and are prone to fugitive leakages (especially in the oil and gas sectors). Until recently, there were no strong incentives to estimate site emissions using dedicated measurements. The reported estimates were usually derived using standard bottom-up products of emission factors times quantity of waste, wastewater, processed gas and/or relatively simple emission models (IPCC, 2013). However, a precise estimate of the methane emissions from these sites based on atmospheric techniques could help their operators to mitigate their emissions as part of their local action plans in the context of climate change. Instantaneous estimates of the emissions through a dedicated measurement campaign can help to detect and provide a useful order of magnitude for sources that are generally poorly known (Yver Kwok et al., 2015). The results from series of campaigns can be extrapolated to estimates for long timescales. However, a continuous monitoring of the emissions with permanent measurements would help to characterise the dependence of emissions on meteorological conditions and on changes in the site processes through time.

Here, we conduct a series of controlled experiments with known emissions of methane from one or two sources and of acetylene from one source, in meteorological and topographical conditions that are compliant with the use of a simple Gaussian plume model. Concentrations are measured through the methane and acetylene plumes at an appropriate distance from the source, as described below. The known emission of methane is used to validate the inversion results, thus assessing the efficiency of our inversion system. In particular, the fit between these results and the actual emissions is compared with the one obtained with the more traditional application of the tracer release technique to demonstrate, under our experimental conditions, the asset of the statistical inverse modelling framework. In Sect. 2, we detail the theoretical framework of the tracer release technique, the local dispersion modelling, the statistical inversion and our concept that combines these different techniques. We also give some practical guidance regarding their application to the monitoring of methane sources and regarding the use of a Gaussian plume model for suitable meteorological and topographical conditions. Then, we describe the configuration and the results of the experiments conducted in this study to evaluate the potential of our approach (Sect. 3). The results and perspectives of the study are discussed in Sects. 4 and 5.

\section{Methods}

\subsection{Instantaneous quantification of pollutant sources using mobile measurements across the atmospheric plumes}

The presentation of the atmospheric monitoring techniques below focuses on their specific configuration for the quasiinstantaneous estimation of emission rates from gas sources within a targeted site. These techniques apply to gases that can be considered inert (non-reactive) on the relevant atmospheric transport and mixing timescales of the experiment. In this case, the representation of atmospheric transport, linking the emissions to the gas concentrations, can be considered linear. Given that these timescales typically correspond to 1 to $10 \mathrm{~h}$, it applies to most pollutants in practice. In this configuration, several times over the course of a few hours and at an appropriate distance from the site, the concentrations are measured along transect lines across the plumes of a gas emitted by the sources. The emission plumes are associated with an increase in the gas concentrations above the background concentration. This background concentration can be characterised by the gas concentrations in the vicinity of the measurement locations that have not been affected by the sources. The increase above the background concentration is proportional to the emission rates (due to the linearity of the atmospheric transport) and it can be identified in the measurements across the plume. Ideally, there should be no other major gas emitter in the vicinity of the targeted site to ensure that, due to the atmospheric diffusion over long distances, the concentrations upwind of the site are relatively constant. Under these conditions the background concentration can be easily characterised.

The choice of the measurement distances should follow several criteria. On one hand, the distance has to be large enough such that the transport from the source to the mea- 
surement is correctly characterised with a local-scale transport model or the proxy from the tracer release. This distance depends on the spread of the single or multiple targeted sources and thus indirectly on the size of the industrial site, but also on meteorological conditions like wind speed and the atmospheric stability. On the other hand, it should be short enough such that the amplitude of the measured concentrations is high enough compared to the measurement and model precision. This criteria essentially depends on the emission rates due to the linearity of the atmospheric transport from the sources: the larger the source, the larger the ratio of the signal to the noise of the measurement, modelling and background and thus the higher the precision of the inversions. Finally, the choice of the distance is constrained by the need to conduct measurements on roads located downwind of the site sources (depending on the specific wind directions during the measurement campaigns) when using instruments on board cars, as in this study.

The simulated relation between the gas emission rates from the single or multiple sources of the site and the atmospheric concentrations relies on knowledge of the location and spread of each source as well as on the proxy of the atmospheric transport. It is linear and expressed by the observation operator $\mathbf{H}$. The relation between the measurement indices of the concentration increase in the emission plumes or "plume indices", hereafter called the observation vector $\boldsymbol{p}$, and the targeted emission rates, called the control vector $f$, is given by the observation equation:

$p=\mathbf{H} \boldsymbol{f}+\boldsymbol{\varepsilon}_{0}$.

$\boldsymbol{\varepsilon}_{0}$ represents the sum of errors from the observation operator in the measurements and in the estimate of the background concentration. The observation vector is derived from the gas concentrations measured for each cross section of the gas plume(s). The atmospheric transport proxy can be derived using the relationship between the known tracer emission collocated with the targeted sources and the tracer concentrations in the tracer release technique (Sect. 2.2) or using a local-scale atmospheric transport model (Sect. 2.3). Inferring gas emissions from gas concentrations implies inverting the atmospheric transport to express $\boldsymbol{f}$ as a function of $\boldsymbol{p}$. If the size of $\boldsymbol{f}$ is the same as that of $\boldsymbol{p}$ (i.e. if the number of plume indices derived from the concentration measurements is set equal to the number of targeted sources) the atmospheric transport matrix $\mathbf{H}$ is a square matrix. If $\mathbf{H}$ is mathematically invertible (i.e. if the problem is not underconstrained due to using indices on plumes that overlap too much) and if the measurement, background and observation operator errors $\boldsymbol{\varepsilon}_{0}$ are ignored, $\boldsymbol{f}$ can directly be derived as $\mathbf{H}^{-1} \boldsymbol{p}$ (Sects. 2.2 and 2.3). If the sizes of $\boldsymbol{f}$ and $\boldsymbol{p}$ differ, or if the measurement, background and observation operator errors $\boldsymbol{\varepsilon}_{0}$ are to be accounted for, statistical inversion approaches can be used to retrieve an optimal estimate of $\boldsymbol{f}$ (Sects. 2.4 and 2.5).

\subsection{The tracer release method}

The tracer release method was developed to quantify pollutant emissions and has already been used in a wide range of studies to estimate the sources of various types of gases such as methane (Babilotte et al., 2010), carbon monoxide (Möllmann-Coers et al., 2002) and isoprene (Lamb et al., 1986). This method consists of releasing a tracer gas with a known rate close to the targeted gas source when this source is clearly localised and of measuring both the targeted and tracer concentrations in sections of the downwind emission plumes. When targeting the total emissions of a site with multiple sources, the tracer release is generally located in the middle of these sources, assuming that the site is seen as a point source from the measurement locations.

When both the released tracer and targeted sources are perfectly collocated and constant in time, the tracer and targeted gas concentrations have the same spatial and temporal relative variations in the atmosphere; i.e. the plumes of the targeted gas and of the tracer have the same structure. In such a configuration, knowledge of the ratio between the tracer plume index $p_{\mathrm{t}}$ and the tracer-controlled emission rate $f_{\mathrm{t}}$ provides a perfect (scalar) observation operator $h$. It thus provides a perfect estimate of the ratio between the targeted gas plume index $p_{\mathrm{m}}$ and the targeted gas emission rate $f_{\mathrm{m}}$. By ignoring the measurement and background errors, the targeted emission rate can be estimated using the following formula:

$f_{\mathrm{m}}=f_{\mathrm{t}} \times \frac{p_{\mathrm{m}}}{p_{\mathrm{t}}}$.

Various types of plume indices $p$ can be used (provided that they are consistently derived for the tracer and targeted gas). The background concentration is generally derived from the measurements before and after crossing the plumes. Then, the plume indices can typically be calculated using the difference between the maximum concentrations (peak heights of the signals) and the background concentration. It can also be derived from the areas between the plume signals and the background concentration (Mønster et al., 2014). When the sources of the released and targeted gases are perfectly collocated and when their emission rates are constant, both of these approaches provide the same result given that the tracer and targeted emission plumes have the same structure. However, if the collocation of both sources is not perfect or if the targeted emissions vary in time, then the shapes of the emission plumes of the released tracer and of the targeted gas can differ. To minimise the impact of this difference, the ratio of the integrated plumes is generally chosen because this index is less sensitive to the impact of thin turbulent structures than the peak height ratio (Mønster et al., 2014). Other indices have also been tested to overcome this issue like the slope of the ratio between the targeted and released concentrations above the background (Roscioli et al., 2015). 
The measurement transects through the emission plumes and the computation from Eq. (2) are generally repeated several times, typically $n_{\text {tr }}=10-15$ times over an hour. The mean and standard deviation $\mathrm{SD}_{\text {tr }}$ of the $n_{\text {tr }}$ different results are used as the best estimate and uncertainty assessment for the source quantification. Note that, strictly speaking, the exact quantification of the uncertainty in the mean estimate should be $\mathrm{SD}_{\mathrm{tr}} / \sqrt{n_{\mathrm{tr}}}$, which will be used here, even though $\mathrm{SD}_{\mathrm{tr}}$ is often used (Yver Kwok et al., 2015). These statistics allow us to account, at least partly, for the potential temporal variations of the emissions, for the measurement and background errors, and for the potential impact of the non-perfect collocation of the sources in the selected measurement transects. In order to strengthen the precision of the best estimate, measurement transects with low correlations between the targeted gas and the released tracer are often ignored. The reason is that such low correlations are related to critical sources of estimation errors. For a range of local meteorological conditions, it can be due to relatively high background and measurement errors compared to the measured signal. It can also be due to a strong difference between the structures of the tracer and targeted gas plumes arising from the fact that the tracer emission is not perfectly colocated with the targeted gas emission.

A mislocation of the tracer source far from the targeted source or its location close to a targeted source with a large spread compared to the distance to the measurements can also generate significant biases in the series of computations. These biases can impact the average estimate of the source without being reflected in the standard deviation of the individual emissions computations or in the correlation between the tracer and targeted gas concentrations. The impact of the mislocation of the tracer source can be decreased by increasing the distance between the sources and the measurements (Roscioli et al., 2015) but the choice of this distance is often constrained by other considerations as discussed in Sect. 2.1. Approaches based on atmospheric transport models have been used to account for errors arising from this mislocation (Goetz et al., 2015).

Moreover, the tracer release technique provides an overall estimate of the emissions of a site. However, when the site has several sources located quite close to each other, it can hardly be used to provide individual estimates of these sources. More specifically, even with the use of different tracer release points, the technique in itself hardly provides solutions to separating overlapping tracer or targeted gas plumes associated with different point sources.

\subsection{Using local-scale transport models}

Many types of transport models are used to simulate the dispersion of pollutants at the local scale, i.e. typically over distances from a few metres to 1 or $2 \mathrm{~km}$, from simple Gaussian models to Lagrangian dispersion and sophisticated CFD (computational fluid dynamics) models that allow tur- bulent patterns to be determined for complex terrain through an explicit representation of reliefs and obstacles (e.g. Baklanov and Nuterman, 2009; Hanna et al., 2011). Beyond the large range of possible model complexity, a common feature of these transport models is their ability to represent sources of any geometry. Therefore, the local-scale transport models allow multiple sources or sources to be addressed with a far more significant spread than proxies based on collocated tracers. In a configuration similar to that of the tracer release technique where concentration measurements are conducted across the $N$ plumes of $N$ targeted gas sources, the local dispersion models can be used to infer the linear relationship between the emission rates and plume indices in each of the measurement transects. The models are run with a zero background concentration unless a strong signal from neighbouring sources outside the targeted site needs to be accounted for, which is not the case in this study.

In practice, for a given measurement transect, simulations with such models for each individual source (ignoring the other ones), with a unitary emission rate can be used to compute each column of the $\mathbf{H}$ matrix in Eq. (1). If the plumes of the $N$ sources do not overlap too much and are all discernable in the measurement transect, an appropriate selection of $N$ plume indices can be used to disentangle these different sources. In such cases, $\mathbf{H}$ is invertible and the derivation of $\mathbf{H}^{-1}$ from matrix $\mathbf{H}$ is straightforward. Consequently, if ignoring the measurement, background and observation operator errors $\boldsymbol{\varepsilon}_{0}, \mathbf{H}^{-1}$ can be directly used for the inversion of the emission of the $N$ different sources as a function of the $N$ plume indices for each measurement transect:

$f=\mathbf{H}^{-1} p$.

As with the tracer release technique, statistics of the results from multiple inversions associated with the different measurement transects can be used to derive a best estimate and its uncertainty. The correlations between the modelled and measured concentrations along the measurement transects can be used to select the most robust inversion cases.

However, the local-scale transport models can bear large uncertainties that are ignored by this inversion. These errors can be directly projected onto the estimate of the emissions through Eq. (3) and thus strongly weaken the confidence in the results. Furthermore, such an inversion can hardly account for the amount of useful information provided by the measurements. Typically, limiting the number of plume indices to the number of targeted sources prevents us from analysing the shape of each emission plume. The shape can indicate the measurement, background and observation operator errors $\boldsymbol{\varepsilon}_{0}$, which can highly impact the inversion results. Finally, with such an inversion, the level of separation between the source plumes has to be evaluated before defining the number of sources that can be inverted separately within a site without solving for an underconstrained problem. When this level of separation is weak, the inversion finds a mathe- 
matical solution to Eq. (3) that can be highly uncertain. The lack of flexibility of such an inversion is thus problematic.

\subsection{Statistical inversion}

The statistical inversion techniques can address the issues associated with Eq. (3) that are discussed above. The Bayesian principle of statistical inversion is to update prior statistical knowledge (i.e. a prior estimate $f^{b}$ and the uncertainties in it) of the emission rates $f$ with statistical information from observations $\boldsymbol{p}$. This update accounts for the statistical uncertainties in the observations (here the measurement and background errors) and in the observation operator $\mathbf{H}$ (Tarantola, 2005). In order to account for several sources within a site, the statistical inversion needs to rely on a local-scale transport model to derive the $\mathbf{H}$ matrix. This theoretical framework allows for a control vector $\boldsymbol{f}$ and an observation vector $\boldsymbol{p}$ taking different sizes into account. All sources can thus be inverted, even if there is not enough information to separate the plumes of some of them. Furthermore, the system can make use of all the information in the measurements to filter the measurement, background and observation operator errors and any signal from the different emissions plumes associated with the different sources.

Assuming that during the measurement campaign the source emission rates are constant, this framework can also be used to assimilate the data from all plume transects to compute the optimal estimate of the emission rates at once. In such a case, the observation vector $\boldsymbol{p}$ gathers plume indices from all the measurement transects and the $\mathbf{H}$ observation operator represents the transport, with various meteorological conditions, from the sources to all the transects. This combination presents advantages over repeating computations for each measurement transect and deriving statistics for the emission estimates out of the ensemble of computations as for the other techniques presented above. In particular, this helps to account for the fact that the sources of errors do not have the same statistical distributions, e.g. the same amplitude for each transect. The previous techniques require a selection of the cases in which the confidence in the observation operator is good enough to enhance the robustness of the average. By assigning model and measurement uncertainties as a function of the measurement transect and/or meteorological conditions, the statistical inversion allows the information from each transect to be weighted differently according to its uncertainty.

The prior estimate of the emission $f^{b}$ has to be independent of the atmospheric observations and can be provided by expert knowledge, emission inventories or process-based models. In practice, it is generally assumed that the uncertainties in $\boldsymbol{f}^{b}$, in the observations $\boldsymbol{p}$ and in the observation operator have unbiased and Gaussian distributions. The prior uncertainty and the sum (henceforth called observation error) of the uncertainties in the observations $\boldsymbol{p}$ (from the measurement and background errors) and on the observation operator
$\mathbf{H}$ are thus characterised by their covariance matrices $\mathbf{B}$ and $\mathbf{R}$, respectively. Following these assumptions, the posterior statistical distribution of the emission rate knowing $f^{b}$ and $\boldsymbol{p}$ is Gaussian and is characterised by its optimal estimate $\boldsymbol{f}^{a}$ and its covariance matrix $\mathbf{A}$ given by equations (Bocquet, 2012):

$$
\begin{aligned}
& \boldsymbol{f}^{a}=\boldsymbol{f}^{b}+\mathbf{B} \mathbf{H}^{T}\left(\mathbf{R}+\mathbf{H B} \mathbf{H}^{T}\right)^{-1}\left(\boldsymbol{p}-\mathbf{H} \boldsymbol{f}^{b}\right) \\
& \mathbf{A}=\left(\mathbf{B}^{-1}+\mathbf{H}^{T} \mathbf{R}^{-1} \mathbf{H}\right)^{-1}
\end{aligned}
$$

The matrix A characterises the unbiased and Gaussian uncertainty in $f^{a}$. If the plume from a source cannot be separated from the other ones, or if the observation errors on the plume indices related to this source are very large, the posterior uncertainty in this source will be large. The $\mathbf{A}$ matrix can thus be used to evaluate the level of constraint on the different sources or on their sum provided by the selection of plume indices and the robustness of the corresponding emission estimates. One difficulty associated with this method is the need to provide a realistic estimate of the observation error statistics, which in practice are difficult to evaluate. Another issue is that, even if the system correctly accounts for the transport modelling errors when well informed about their statistics, it will derive very uncertain emission estimates if these transport errors are large.

\subsection{A statistical inversion based on tracer release and local-scale transport modelling}

Here, we propose a new concept for the estimation of the gas emission rates combining the tracer release method, localscale transport modelling and a statistical inversion framework to overcome the issues associated with these different approaches and tools as discussed above. The basis of this new concept is the statistical inversion framework, assimilating the plume indices from all measurement transects altogether, where the $\mathbf{H}$ matrix is derived from local-scale transport model simulations for each point or spread source of a targeted site and each measurement transect.

The main idea is to use the very accurate information on the atmospheric transport in the area of interest from the tracer release method to adjust parameters of the local-scale transport models and to assess the statistics of the transport errors. The optimised transport model and the statistics of the transport errors are then used for the configuration of the observation operator and of the observation errors in the statistical inversion system outlined in the previous section. The optimisation of the transport model parameters can rely on a range of methods, from a simple comparison between an ensemble of tracer simulations with different sets of parameters and the tracer measurements to complex tracer data assimilation.

The statistics of the misfits between the tracer measurements and the model-based concentrations when using the optimal transport model configuration are used to set up the 
covariances of the observation (measurement, background and observation operator) errors $\mathbf{R}$. This requires the conversion of the error statistics for the tracer gas into statistics of the errors for the targeted gas. Therefore, the statistics of the variability of the measured tracer and targeted gas concentrations are used to normalise the transport errors for the two species as relative errors, and the assumption is made that the relative transport errors are the same for both species.

Model parameters and/or characterisation of the transport errors can be optimised for each individual crossing of the plume or for all plume crossings together. The use of a specific optimisation of the model for each plume crossing may be preferable if the local meteorological conditions evolve rapidly. Using general statistics of the tracer model-data misfits from all plume crossings would prevent the transport error from being weighted and thus the information for each plume crossing depending on the modelling skills. Deriving different transport errors for each plume crossing requires the extrapolation of the single set of tracer model-data misfits into statistics for each plume crossing. These different options need to be chosen depending on the experimental case.

In order to investigate the potential of this approach in a first real test case, we propose a relatively simple practical implementation using a Gaussian transport model. CFD models remain sophisticated tools. The choice of a Gaussian plume model is more appropriate for the introduction and first test of our concept but we are aware that it restrains the range of situations that can be investigated.

\subsection{Practical implementation for the monitoring of the methane sources using a Gaussian plume model and acetylene as tracer}

\subsubsection{The Polyphemus Gaussian plume model}

Gaussian plume models provide a stationary and average view of the pollutant plumes driven by meteorological conditions that are stationary in time and homogeneous in space. This is a decent approximation for the dispersion over 12 min (i.e. the typical timescale associated with our experiments) and an area of approximately $1 \mathrm{~km}^{2}$ when the wind speed is relatively high. These models cannot account for the effects of complex local topography and buildings. However, they are suitable for many configurations of industrial sites located in nearly flat suburban to rural areas, and they are easily set up and applied for the simulation of local-scale transport.

In this study, the Gaussian plume model of the Polyphemus air quality modelling system (Mallet et al. (2007) http: //cerea.enpc.fr/polyphemus/) is used because it has been proven to be adapted for estimating gas emissions from local sites (Korsakissok and Mallet, 2009). Gaussian plume models are based on a simple formula that provides the concentration of the pollutant at a location generated by a point source depending on the weather conditions. The Gaussian plume formula is expressed as

$$
\begin{aligned}
& C(x, y, z)=\frac{Y}{2 \pi \sigma_{y} \sigma_{z} \bar{u}} \exp \left(-\frac{\left(y-y_{\mathrm{s}}\right)^{2}}{2 \sigma_{y}^{2}}\right) \\
& \times\left[\exp \left(-\frac{\left(z-z_{\mathrm{s}}\right)^{2}}{2 \sigma_{z}^{2}}\right)+\exp \left(-\frac{\left(z+z_{\mathrm{s}}\right)^{2}}{2 \sigma_{z}^{2}}\right)\right],
\end{aligned}
$$

where $C$ is the concentration of the pollutant at a location of coordinates $(x, y, z), Y$ is the source emission rate, and $\bar{u}$ is the wind speed. In this formula, the $x$ axis corresponds to the wind direction, $y_{\mathrm{s}}$ is the pollutant source ordinate (for a single source usually set to zero) and $z_{\mathrm{s}}$ is the release height above the ground. As both studied gases are poorly soluble and chemically inert for the considered dispersion timescale, it is appropriate to neglect the mass loss due to dry deposition and assume a total reflection from the ground as expressed by the last exponential term in the equation. The values $\sigma_{y}$ and $\sigma_{z}$ are the horizontal and vertical Gaussian plume standard deviations and characterise the atmospheric conditions during the measurements. The modelled concentrations are strongly dependent on these two parameters. Within the Polyphemus system, several ways in which to parameterise these constants are available: the Doury formulas (Doury, 1976), the Pasquill-Turner formulas (Pasquill, 1961) and the Briggs formulas (Briggs, 1973).

The parameterisation according to Briggs is the most flexible one. This parameterisation considers the stability of the atmosphere via the six classes of the Pasquill classification from A (extremely unstable) to F (extremely stable) by taking into account wind speed and solar irradiance. It also considers the type of environment with an urban mode when the emission source is surrounded by buildings and a rural mode for isolated sites (by changing the roughness factors). The standard deviations with this parameterisation are given by the following equations:

$\sigma_{y}=\frac{\alpha x}{\sqrt{1+\beta x}}$ and $\sigma_{z}=\alpha x(1+\beta x)^{\gamma}$,

where $x$ is the downwind distance from the source and $\alpha$, $\beta$ and $\gamma$ are coefficients that are dependent on the stability classes. All these coefficients can be found in Arya (1999).

Different source spatial extensions can also be created in this model. However, its configuration imposes the emission $f_{i}$ of a given source to be spread homogeneously over its extension. The Gaussian plume model cannot represent the instantaneous turbulent structures at fine spatial and temporal scales but rather represents a time-averaged view of a plume. Therefore, it is expected that, by using a high number of measurement transects, the Gaussian plume model should appropriately depict an average plume and that the transient turbulent patterns in the measurements would generate noise in the emission estimates without biasing it. 
Table 1. Weather conditions during the four tests and configuration of the observation vector for the statistical inversion.

\begin{tabular}{|c|c|c|c|c|c|c|}
\hline \multirow[b]{2}{*}{$\begin{array}{l}\text { Trace gas } \\
\text { configuration }\end{array}$} & \multicolumn{3}{|c|}{ Weather conditions (avg.) } & \multirow{2}{*}{$\begin{array}{r}\text { Total } \\
\text { number of } \\
\text { transects }\end{array}$} & \multirow{2}{*}{$\begin{array}{r}\text { Number } \\
\text { of selected } \\
\text { transects }\end{array}$} & \multirow{2}{*}{$\begin{array}{l}\text { Configuration of the } \\
\text { observation vector for the } \\
\text { statistical inversion }\end{array}$} \\
\hline & $\begin{array}{r}\text { Temperature } \\
\left({ }^{\circ} \mathrm{C}\right)\end{array}$ & $\begin{array}{c}\text { Wind } \\
\text { direction }\end{array}$ & $\begin{array}{l}\text { Wind speed } \\
\quad\left(\mathrm{m} \mathrm{s}^{-1}\right)\end{array}$ & & & \\
\hline Configuration 1 & $9.9 \pm 0.3$ & $\mathrm{~N}$ & $3.2 \pm 0.6$ & 29 & 11 & Integration of the entire plume \\
\hline Configuration 2 & $9.2 \pm 0.1$ & $\mathrm{~N}$ & $3.7 \pm 0.8$ & 20 & 9 & Integration of the entire plume \\
\hline Configuration 3 & $8.4 \pm 0.8$ & $\mathrm{~N}$ & $2.5 \pm 0.7$ & 35 & 10 & Integration of the entire plume \\
\hline Configuration 4 & $11.3 \pm 0.3$ & NE & $2.0 \pm 0.7$ & 40 & 8 & Integration of slices of the plume \\
\hline
\end{tabular}

\subsubsection{Adjustment of the stability class underlying the Briggs parameters and estimate of the Gaussian model errors using the tracer data}

The application of the new statistical inversion strategy described in Sect. 2.5 in connection with the Polyphemus Gaussian transport model relies on the optimisation of the stability class underlying the Briggs parameters and of the plume direction as a function of the tracer measurement transects. Since wind was measured directly in our experiments, a correction of the Gaussian plume direction should not be needed, but Sect. 3.2 will describe practical issues which require a correction.

For each measurement transect, the method consists of running multiple model tracer simulations with different stability classes. They are all forced with the known tracer emission rate. The model plume direction is adjusted so that the measured and simulated plumes are aligned. The stability class with the simulation of the tracer concentrations that best fits the measurements is taken as the optimal one. The fit is quantitatively checked for the plume indices chosen for the definition of $\boldsymbol{p}$, but it is also checked in a qualitative way by analysing the shape of the modelled and measured signals. The estimate of the Gaussian model errors is based on statistics of the misfits between the modelled and measured tracer plumes indices.

\subsubsection{Monitoring of the methane sources using controlled release of acetylene}

This method is tested for the quantification of methane sources using acetylene as a tracer gas. The lifetimes of methane and acetylene are approximately 10 years and 24 weeks (Logan et al., 1981). Both of these gases can be considered inert at the timescale corresponding to the time between the release of molecules at the source and the measurement of concentrations downwind in the plume.

In this study, the methane and acetylene concentrations are measured in a continuous manner along a line crossing the emission plumes using a sensitive analyser placed in a car. Our preliminary analysis shows that we obtain satisfying results when concentrations are typically measured at distances of 100 to $1000 \mathrm{~m}$ from methane sources of 1500 to
$100000 \mathrm{gCH}_{4} \mathrm{~h}^{-1}$ and spread within an area of $100 \times 100$ to $500 \times 500 \mathrm{~m}^{2}$.

\section{Evaluation of the concept with controlled release experiments}

\subsection{General principle of the controlled experiments}

The following sections describe the experiments under controlled conditions for both acetylene and methane, which are used to evaluate the statistical inversion framework detailed in Sect. 2.6 and more generally to give insights into the potential of the approach proposed in this study in Sect. 2.5. A campaign was organised over 2 days in March 2016 at the Laboratoire des Sciences du Climat et de l'Environnement (LSCE) in France (longitude: 48.708831 latitude: $2.147613^{\circ}$, altitude a.s.l.: $163 \mathrm{~m}$ ). The experimental conditions were selected to be favourable for the use of a Gaussian plume model to simulate the atmospheric transport. One or two methane sources and one acetylene point release were generated with cylinders in the car park of the LSCE, which is located in a rural area in the southern region of Paris. The topography of this area is very flat, and only few low buildings can potentially influence the atmospheric transport from the car park to the road where the concentrations are measured. This road is located approximately $150 \mathrm{~m}$ away from the controlled sources. No major methane or acetylene sources in the vicinity of the LSCE could disturb the measurements. Each measurement day was selected by taking the weather forecast into account and choosing days with a strong enough wind from the north to be able to measure the emissions from the car park on the measurement road. The average weather conditions of each measurement series are summarised in Table 1.

During this campaign, the methane and acetylene sources were dispersed in four different configurations to estimate the accuracy of the proposed method and the uncertainties depending on whether the tracer gas is perfectly collocated with the methane source or not. For each configuration, the methane and acetylene emission plumes were crossed 20-40 times (see Table 1), and each series of crossings occurred on the same day on a timescale of 1-2 h. The observed increases in the acetylene and methane concentrations within 
the plumes ranged between 3-15 and 50-500 ppb. Configurations 1,2 and 4 were tested in the afternoons between 13:30 and 16:30 UTC, while configuration 3 was tested in the morning between 10:00 and 12:00 UTC (see Fig. 1).

The following sections describe the different components of the experimental and modelling systems used for the inversion of the methane sources and the results from both the tracer release technique and the combined statistical approach. These results are compared with the known methane emission rate to test the ability of each method to estimate the emissions. Statistics of uncertainties are also derived for the two methods based on the statistical frameworks described in Sect. 2 but also based on Observing System Simulation Experiments (OSSEs) with pseudo-data.

\subsection{Analytical equipment}

Downwind gas concentrations were measured using a G2203 cavity ring-down spectrometer (Picarro, Inc., Santa Clara CA), which continuously measures acetylene $\left(\mathrm{C}_{2} \mathrm{H}_{2}\right)$, methane $\left(\mathrm{CH}_{4}\right)$ and water vapour $\left(\mathrm{H}_{2} \mathrm{O}\right)$. Based on infrared spectroscopy, the high precision of the system (precisions of $3 \mathrm{ppb}$ and $<600 \mathrm{ppt}$ for methane and acetylene, respectively, on a $2 \mathrm{~s} \mathrm{interval})$ is due to its very long path length $(\simeq 20 \mathrm{~km})$ and the small size of its measurement cell $(<35 \mathrm{~mL})$. Mobile measurements with this instrument have already been successfully taken and published in previous studies (Mønster et al., 2014; Yver Kwok et al., 2015), demonstrating the potential of this method. The measurement error encompasses the precision stated above but also the fact that the acetylene and methane are not measured at the exact same time and frequency. Indeed, acetylene is measured every second while methane is measured every other second. At the scale of our measurement (less than a minute to cross a plume), this can impact the error significantly.

Before the experiment, the instrument has been tested in the laboratory. It showed good linearity over a large range of mixing ratios and high stability over time with small dependency on pressure and temperature. To control for a drift, we measured a gas with a known mixing ratio (calibrated with a multi-point calibration in the laboratory) before each series of measurements in order to ensure the good analytical performance of our instrument. Moreover, in the tracer release method and the combined approach presented in this study, we are interested in the increase in concentrations due to the tracer and targeted point sources above the background signal (i.e. the plume indices) more than in the absolute value of the measurements. Thus, an offset of the measured concentrations will not impact our estimates.

During the field campaign, wind speed and direction were taken from the meteorological station installed on the roof of the nearby laboratory at about $7 \mathrm{~m}$ high. The mobile system was set up in a car and powered by the car's battery. The air sampler was placed on the roof at approximately $2 \mathrm{~m}$ above the ground with a GPS (Hemisphere A21 Antenna) to provide the location of the measurements. The sampled air was sent into the instrument by an external pump system, allowing an inlet lag between the sample inlet and the measurements of less than $30 \mathrm{~s}$. This more or less constant inlet lag introduced a spatial offset when comparing the measured and modelled tracer or methane concentrations. This spatial offset is the same for methane and acetylene and is characterised by the comparison between the modelled and measured acetylene plumes. In our combined statistical approach, it is thus well accounted for when comparing the modelled and simulated methane plumes thanks to the correction of the Gaussian plume direction according to the acetylene data. Therefore, this offset is ignored hereafter.

\subsection{Tracer and target gas release}

Acetylene is commonly used as a tracer. Due to its low concentration in the atmosphere $(\simeq 0.1-0.3 \mathrm{ppb})$, any release is easily detected. Acetylene also has the benefit of being relatively inert, and thus, negligible loss during the transport process is expected (Whitby and Altwicker, 1977). Other gases are suitable as tracers, such as $\mathrm{SF}_{6}$, but acetylene is preferred because it is not a greenhouse gas. However, due to its flammability, its use requires specific precautions.

An acetylene cylinder $(20 \mathrm{~L})$ containing acetylene with a purity $>99.6 \%$ was used as the tracer source. A methane cylinder $(50 \mathrm{~L})$ with a purity of $99.5 \%$ was used for the controlled methane release. The flows of both gases were controlled by a $150 \mathrm{~mm}$ flow meter (Sho-rate, Brooks) which was able to measure fluxes between 0 and $1500 \mathrm{~L} \mathrm{~min}^{-1}$. The different acetylene and methane emission rates were checked by weighing the cylinders before and after each test and timing the release duration. The flow rate calculated with the mass difference was systematically in good agreement with the flow rate reading on the flow meter, since their relative difference was between 1 and $3 \%$. Therefore, we believe that there was no significant variability in the acetylene and methane release during our experiments. The amount of acetylene emitted was adjusted such that its emission plume can be detected on the roads where the measurements were taken while keeping it at the lowest rate possible to limit the risks associated with its flammability. In this study, we used emission rates from 65 to $90 \mathrm{~g} \mathrm{~h}^{-1}$ for acetylene. During the measurement campaign, the cylinders were attached with straps to a fixed frame to avoid any accidents.

\subsection{Tested configurations of the gas releases}

This section details the four configurations used during this campaign (Fig. 2). The first configuration consisted of a collocated emission of acetylene and methane. This configuration enabled us to estimate the accuracy of the method and our system under optimal conditions. One cylinder of methane and one cylinder of acetylene were placed in the car park and connected together by a tube with a length of a few 

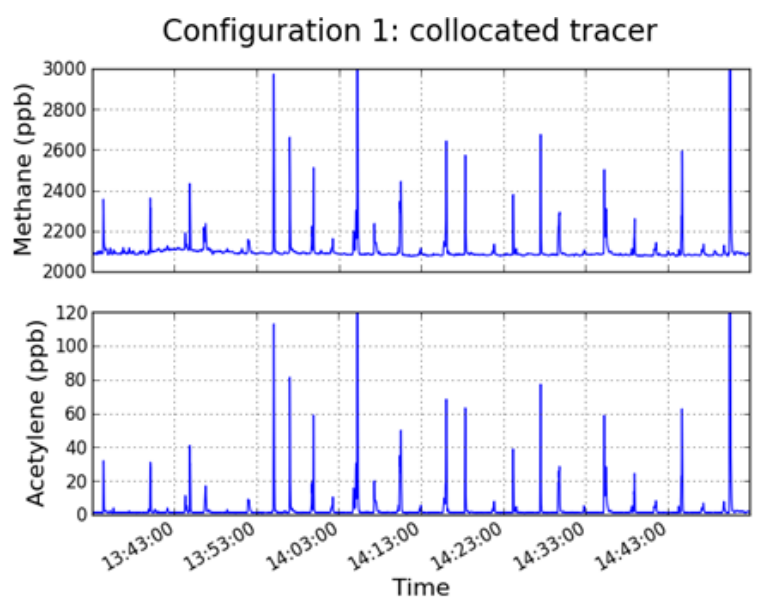

Configuration 3: lateral tracer
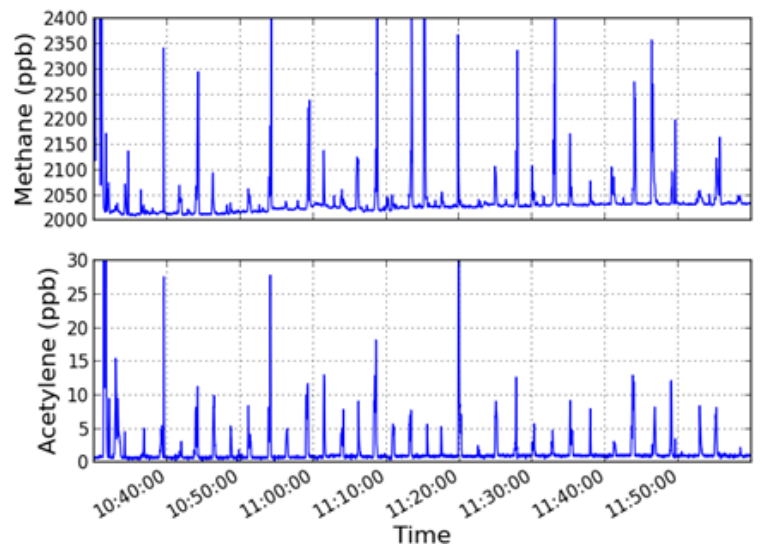

Configuration 2: upwind tracer
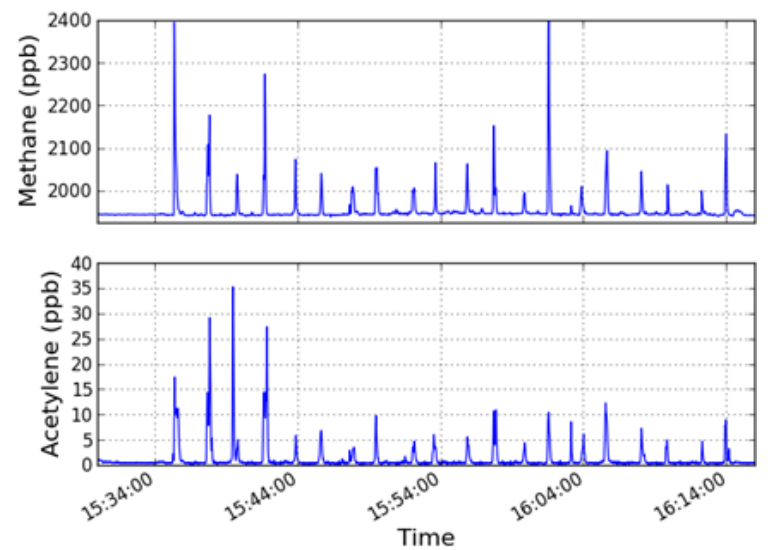

Configuration 4: multiple sources
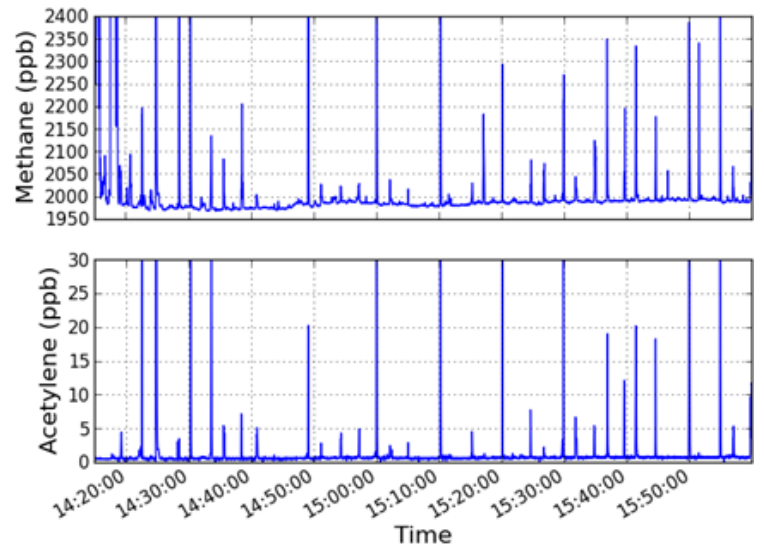

Figure 1. Concentrations of methane and acetylene during the four tracer release experiments.

metres. This system aimed to ensure optimal mixing of both gases and was designed to be as close as possible to the ideal situation in which methane and acetylene are emitted at the same location and under the same conditions. In principle, under such conditions, the tracer-concentration-to-emission ratio should provide a perfect proxy of the methane transport and the tracer release technique should provide better estimates than the statistical inversion that relies on an imperfect but optimised modelling of the methane plume. Still, both techniques should be hampered by measurement and background errors.

In reality, at industrial sites, methane source locations are not always well known, or it may be difficult to access these sources and place a tracer cylinder next to them. The second and third configurations tested the impact of non-collocated emissions of tracer and methane. To represent this situation, one cylinder of methane and one cylinder of acetylene were used, and the methane cylinder was moved (i) approximately $60 \mathrm{~m}$ downwind from the acetylene bottle location (second configuration) and (ii) approximately $35 \mathrm{~m}$ laterally from the wind direction (third configuration). During these two ex- periments, the wind was blowing from the north; i.e. it was perpendicular to the measurement transects along the road, south of the sources.

Finally, within real industrial sites, several sources of methane may be encountered. The fourth configuration tested the influence of having two methane sources on the estimation of their fluxes when one tracer source is used. With this configuration, we also evaluate the ability of the combined statistical approach to estimate the emissions for each individual methane source. For this purpose, a system of two tubes was connected to the methane cylinder, splitting its exhaust into two locations approximately $35 \mathrm{~m}$ apart. During this experiment, the wind was blowing from the northeast, i.e. it was not perpendicular to the measurement transects along the road. The acetylene cylinder was collocated with one of the exhausts.

The advantage of the combined method proposed in this study over the traditional tracer release technique (which relies on the collocation of the target and the tracer gas sources) to infer the total emissions from a site should be revealed in the second and fourth experimental configurations. In homo- 


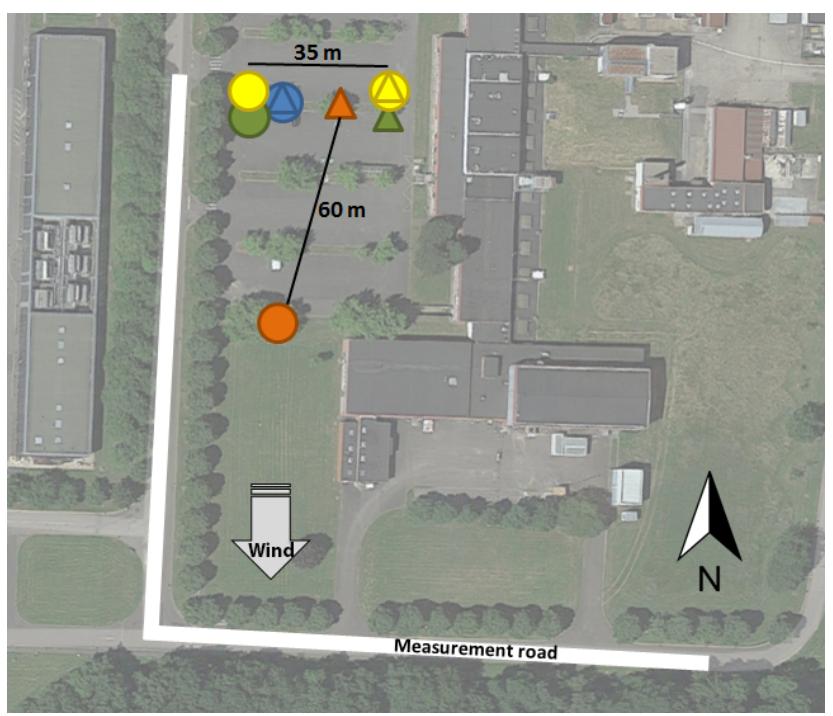

Figure 2. The four tracer release configurations tested. Triangles represent the tracer source locations, and the circles mark methane sources. Each colour represents a configuration: blue is configuration 1 (collocated tracer), red is configuration 2 (upwind tracer), green is configuration 3 (lateral tracer) and, yellow is configuration 4 (multiple sources).

geneous meteorological conditions, and when the wind direction is perpendicular to the measurement transects, a shift of the methane sources in a direction perpendicular to the wind and parallel to the measurement transects should only result in a shift of the emission plumes along the measurement transects. It should not impact the plume indices from the measurement transects and thus the results from the tracer release technique. Therefore, under idealistic conditions, in the third experimental configuration, the tracer release technique should still provide better estimates than the combined approach. However, in practice, during experiments with the third emission configuration, neither the shift between the cylinders nor the measurement transects (along the slightly curvilinear road) were perfectly perpendicular to the wind direction, and they were not perfectly parallel between them. Therefore, the combined approach also has the potential to yield better estimates in this configuration. Finally, it can provides estimate for both sources in the fourth configuration, while this cannot be achieved with the tracer release technique in our experimental framework due to the strong overlapping of the plumes from the individual sources (see Sect. 3.8).

The time series of acetylene and methane measurements for each tracer release experiment are shown in Fig. 1.

\subsection{Definition of the background concentration and of the plume indices}

In this study, two different definitions of the plume indices are used to build the observation vector $\boldsymbol{p}$, but they are both based on the integral of areas between the concentrations within the plumes and the background concentration.

The portions of plume concentrations and of background concentrations in the measurement transects are defined "by eye". The portions of background concentration are restricted to $\simeq 5 \mathrm{~s}$ before and after the plumes. In many cases, the increase in the concentrations due to the plumes is clear and the portions of plume and background concentration are easy to define. However, in other cases the background variations near the plumes and the turbulent patterns at the edge of the plumes can have comparable amplitudes so that defining these portions is more difficult (Fig. 1). For each plume, the background concentration value used to compute the plume index is taken as the average concentration over the background portions of the transect.

When we investigate the tracer data or when we estimate the emission rate of a single source of methane, i.e. in configurations 1, 2 and 3, and in configuration 4 for the tracer release technique only, the plume indices are defined as the integral over the entire plume of the concentrations above background. In this case, the observation scalar $p$ (when applying the tracer release technique to each transect) or vector $\boldsymbol{p}$ are denoted $\boldsymbol{p}^{\text {ent }}$ and $\boldsymbol{p}^{\text {ent }}$ when conducting the combined statistical inversion by gathering data from all transect into a single vector.

When we estimate the emission rates of the two sources of methane with the combined approach in configuration 4 , the portion of observed methane and acetylene increase within the plumes is divided into five slices of equal time (and identical for methane and acetylene). For each slice of a given transect, an index $p^{\text {slc }}$ is defined as the integral of the concentrations above the background in this slice. The observation vector $\boldsymbol{p}^{\text {slc }}$ gathers all these indices.

\subsection{Optimisation of the Gaussian plume model parameters}

In the Polyphemus Gaussian plume model, the definition of the plume indices is consistent with the one in the measurements, and in particular it follows the same definition of the plume portions or slices along the measurement transects.

For each measurement transect, the optimisation of the stability class underlying the Briggs parameterisation of this model is based on the fit to the acetylene plume index only. Since no measurements of solar irradiance were available, comparing the selected stability class to the theoretical one is not possible. According to the table of Pasquill, which is used for the Briggs parameterisation, there are three stability classes that correspond to the 2 to $4 \mathrm{~m} \mathrm{~s}^{-1}$ measured wind speed during our experiments: the classes A and B and C. 
However, for a given wind speed, there is only two choices, $\mathrm{A}$ and $\mathrm{B}$, or $\mathrm{B}$ and $\mathrm{C}$. We have verified, for each measurement transect, that the selected stability class is consistent with these two theoretical options. Choosing one over the other can modify the simulated plume indices by a factor 2 to 3 .

We also checked for each measurement transect that the model error is not too large. In some cases, the model cannot reasonably reproduce the observations due to the presence of pronounced turbulent structures or due to transport conditions that are extremely unfavourable for the model (due to swift wind change or low wind conditions). In such situations, there is no Briggs stability class that allows the model to approximately fit the acetylene plume index. Finally, we decided to remove transects from the analysis when the relative error between the modelled and measured acetylene plume indices was higher than $70 \%$. This value of $70 \%$ is an empirical choice corresponding to very large modelling errors. All cases kept for the analysis had relative uncertainties well below this $70 \%$ threshold. In theory, the strategy of computing the statistics of the model error as a function of such misfits should ensure that the weight given to these transects in the inversion is low. However, in practice, we conservatively prefer to remove transects for which the confidence in the model is extremely low. This evaluation leads us to ignore $30 \%$ of measurement transects when applying the combined statistical approach.

Figure 3 illustrates the results of the model parameterisation selection. In this example, which corresponds to the fifth transect of the measurements for configuration 2 when the wind speed was $2.9 \mathrm{~m} \mathrm{~s}^{-1}$, the tracer concentrations modelled with the stability class B best fit the measured concentrations, which are represented in black.

\subsection{Estimation of the biases of the tracer release method due to the mislocation of the tracer with theoretical model experiments}

When using the tracer release technique, defining the optimal estimate of the emissions and the uncertainty in the estimate from the $n_{\mathrm{tr}}$-selected transects as the average estimate from the application of Eq. (2) and using $\mathrm{SD}_{\mathrm{tr}} / \sqrt{n_{\mathrm{tr}}}$, fully ignores any potential bias in the method. However, in our experiments, the mislocation of the tracer emission does not only generate random errors that are caught by the variations of the results between the different measurements transects. It also has a strong potential to generate a bias in the computations, since the measurements are taken in a relatively narrow range of positions south of the sources. Such a problem applies to many of the tracer release experiments in which the measurements are always taken from the same area (e.g. due to needing to use roads).

Here, we use OSSEs (Rayner et al., 1996; Chevallier et al., 2007) with the Gaussian plume model. Its stability class is optimised with the tracer data to estimate the bias that can

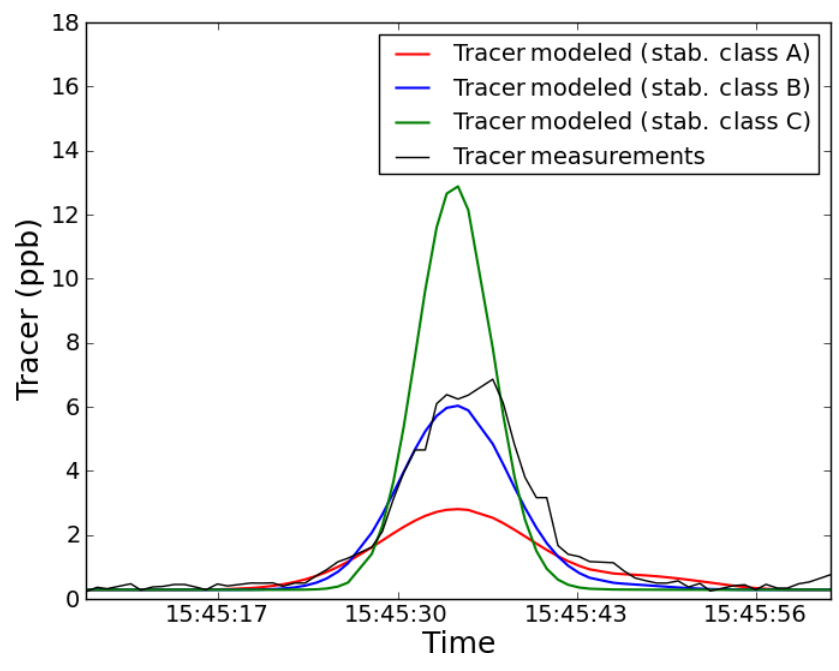

Figure 3. Example of the Briggs parameterisation selection with the acetylene data for peak 5 of configuration 2 . The measured concentrations are presented in black, and the modelled concentrations with different stability classes are shown in colours.

arise from the spatial offsets between the tracer and methane sources. The bias estimates will be used to complement the assessment of uncertainty in the results from the tracer technique, except for the first configuration of the experiments, for which there is no offset between the methane and acetylene sources. As discussed in Sect. 3.4, the lateral (i.e. orthogonal to the wind direction and parallel to the road) offsets between the methane and tracer sources in the third experimental configurations are expected to have a relatively weak impact on the tracer release computations. There should be a far larger bias associated with the downwind shift of the unique methane sources in the second configuration and with the complex shift of one of the methane source when the wind was not blowing perpendicular to the measurement transects in the fourth experimental configuration.

In the OSSEs, we assume that the true methane and acetylene emission rates are those used for the experiments with real data. The synthetic methane and acetylene concentrations are simulated with the Gaussian plume model forced with these emission rates and similar weather conditions to those during the campaign. The corresponding emission plume transects for both gases are extracted along the same paths as during the campaign. Finally, Eq. (2) is applied with the acetylene and methane plume indices from these simulations and the acetylene emission rate, and the resulting methane emission rate is compared to the actual one. The comparison provides a direct estimate of the bias associated with a spatial offset between the acetylene and methane sources, since in these computations (i) stationary conditions are implicitly assumed, (ii) the same model configuration is used to simulate the acetylene and methane concentrations, and (iii) we ignore the background and measurement errors. 


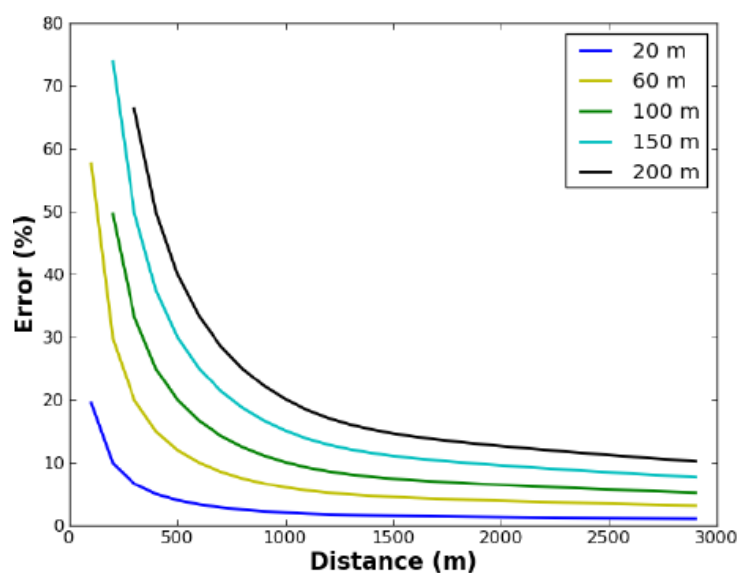

(a) Downwind tracer shift

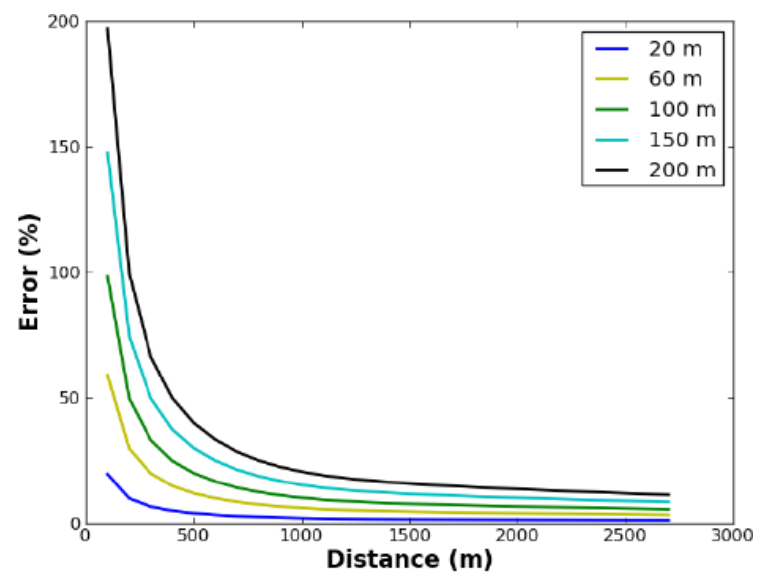

(b) Upwindtracer shift

Figure 4. Error in plume estimation with the tracer method depending on the measurement distance to the methane source and a shift of 20 , 60,100150 and $200 \mathrm{~m}$ of the tracer location relative to the methane source using our Gaussian plume model.

In the following, we characterise the biases by their absolute value and the fraction of the actual source that they represent. The bias is estimated to be $69 \%$ for configuration 2 , $12 \%$ for configuration 3 and $56 \%$ for configuration 4 . Considering the amplitude of these errors, we can expect that our combined statistical approach has a high potential for providing better estimates than the tracer release approach for configurations 2, 3 and 4 .

Additional OSSEs are conducted to better characterise the biases as a function of the upwind or downwind shifts of the tracer source compared to the targeted source and as a function of the distance between the sources and the measurement locations. They correspond to the theoretical experimental configurations with one methane and one acetylene source only, and they use northern wind conditions as were measured during the first experimental configuration. Upwind and downwind offsets from 20 to $200 \mathrm{~m}$ between the methane and tracer sources are tested with OSSEs, with hypothetical measurement transects perfectly orthogonal to the plumes (wind) directions at different distances from 100 to $2750 \mathrm{~m}$ from the methane source. The corresponding estimates of biases are presented in Fig. 4, with the results for the downwind and upwind shifts of the acetylene source provided in Fig. 3a and b.

When the tracer is released upwind of the methane source, the emission rate is overestimated because of the vertical atmospheric diffusion, which makes the integral of the released tracer concentrations through the emission plume near the ground lower than if both sources were collocated. The opposite occurs if the released tracer is placed downwind of the methane emission location. When the tracer source is either upwind or downwind of the methane source by more than $100 \mathrm{~m}$ and the measurements are taken at less than $300 \mathrm{~m}$, the bias exceeds $40 \%$. The biases due to upwind shifts are generally similar to the biases due to downwind shifts over the same distances. When the measurement distance increases, the impact of the shift between the sources decreases. When the measurements are taken at more than $1200 \mathrm{~m}$, the bias is less than $20 \%$. However, at such distances, with the emission rates used in our experiments, the signal-to-measurement and background noise ratio would likely be too small for our instruments to derive precise estimates of the emissions.

\subsection{Tracer release method estimates}

Figure 5 presents one example of the measured acetylene and methane cross sections used for calculating the methane emission rate for each campaign. For the first series, both the acetylene and methane profiles are similar due to the collocation and the mixing of the sources, but we can still observe a significant difference between both emission plumes due to measurement and background errors. The shift between the sources is reflected by a smaller relative amplitude and a higher relative width of the acetylene plume compared to the methane plume in configuration 2 than in configuration 1 and by a lateral shift of the acetylene plume compared to the methane plume in configuration 3 . The two overlapping methane emission plumes, one superimposed with the acetylene plume, can be distinguished in the fourth configuration.

In this section, the uncertainties in the optimal (i.e. average) estimates of the sources are characterised by the random uncertainty which is given by (i) the variations of the results between the measurement transects, $\mathrm{SD}_{\mathrm{tr}} / \sqrt{n_{\mathrm{tr}}}$, (ii) the bias due to the mislocation of the tracer (see Sect. 3.7 above), and (iii) the standard deviation of the total uncertainty being taken as the root sum square of these two terms. Table 2 lists the estimated methane emission rates and the methane emission rates actually released for each tested configuration.

These results confirm that the estimates closest to the actual methane rates are obtained for the first and the third con- 

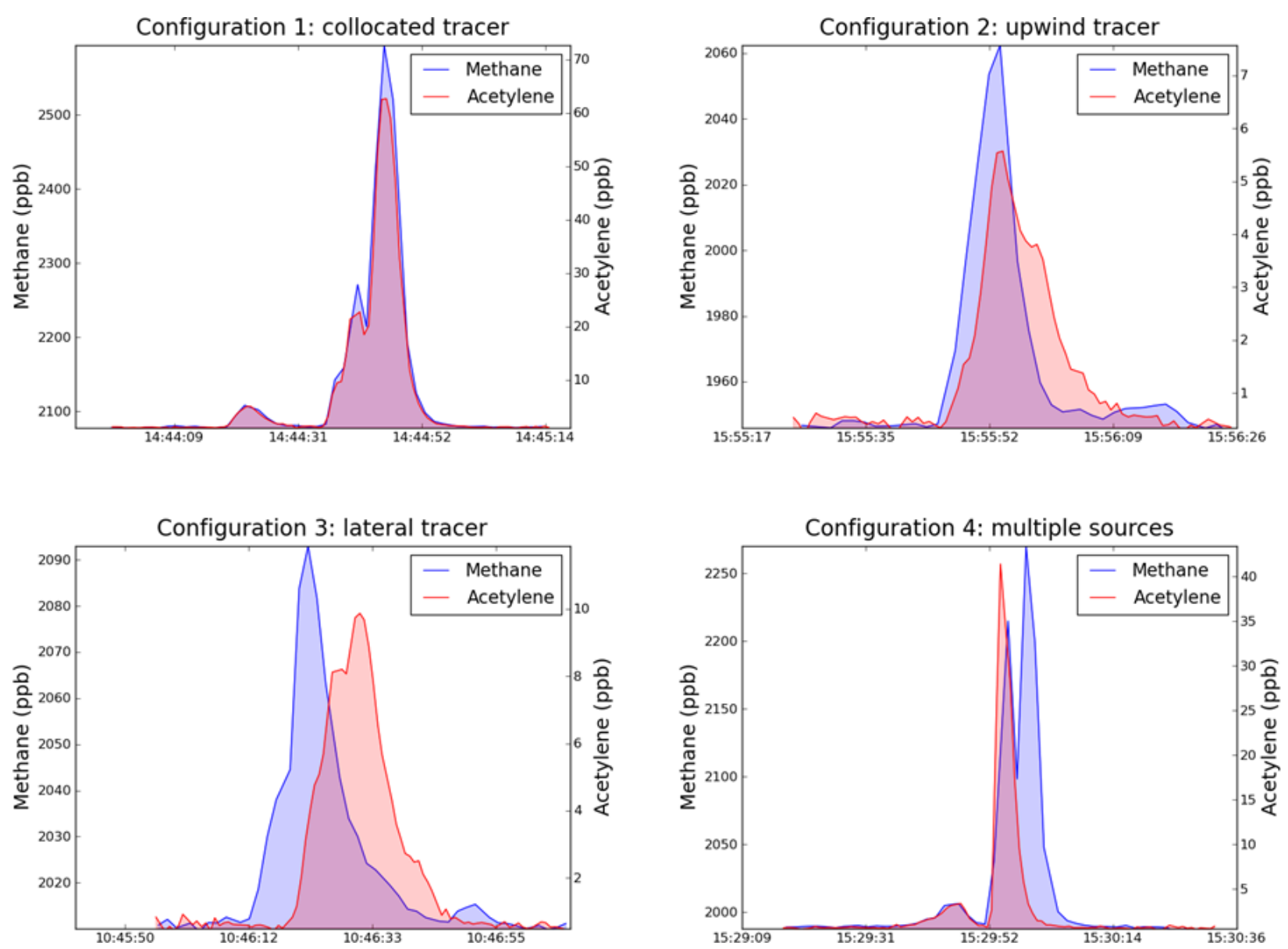

Figure 5. Examples of cross sections of the measured emission plumes of acetylene and methane (in red and blue) for each configuration.

Table 2. Methane emission rates of the different controlled release configurations estimated with the different approaches and methane fluxes actually emitted during these tests. The uncertainties given with the tracer release method are detailed as follows: standard deviation of the random uncertainty derived from the variability in the results from one transect to the other one (bias due to the mislocation of the tracer; total uncertainty).

\begin{tabular}{lcccc}
\hline & $\begin{array}{c}\text { Configuration 1 } \\
\text { (collocated tracer) }\end{array}$ & $\begin{array}{c}\text { Configuration 2 } \\
\text { (upwind tracer) }\end{array}$ & $\begin{array}{c}\text { Configuration 3 } \\
\text { (lateral tracer) }\end{array}$ & $\begin{array}{c}\text { Configuration 4 } \\
\text { (multiple sources) }\end{array}$ \\
\hline Controlled methane release $\left(\mathrm{g} \mathrm{h}^{-1}\right)$ & $382 \pm 7$ & $428 \pm 7$ & $360 \pm 7$ & $482 \pm 7$ \\
\hline $\begin{array}{l}\text { Tracer release method estimates }\left(\mathrm{g} \mathrm{h}^{-1}\right) \\
\text { Relative difference to the control release }(\%)\end{array}$ & $434 \pm 23(0 ; 23)$ & $564 \pm 120(295 ; 415)$ & $321 \pm 51(43 ; 94)$ & $804 \pm 160(270 ; 430)$ \\
67 & 32 & $386 \pm 2$ & $462 \pm 34$ \\
Combined approach estimates $\left(\mathrm{g} \mathrm{h}^{-1}\right)$ & $441 \pm 6$ & $358 \pm 2$ & 4 & 4 \\
Relative difference to the control release $(\%)$ & 15 & 16 & 767 \\
\hline
\end{tabular}

figurations with relative differences of 14 and $11 \%$. However, surprisingly they are slightly higher for the first configuration than for the third one. Furthermore, these errors are relatively high for the tracer release technique. They are mainly due to the variations in the background concentrations for methane, but also in some cases for acetylene. For example, the methane background can range between 2079 and 2099 ppb from one crossing to another for the first con- figuration or between 2012 and 2031 ppb between transects for the third configuration. Moreover, the standard deviations within the background portions used to compute the background concentration can reach $9 \mathrm{ppb}$ for methane and $1 \mathrm{ppb}$ for acetylene. These variations characterise the level of uncertainty in the background concentration and they are significant compared to the amplitude of the plumes. The measurement errors associated with the lag time between the methane 

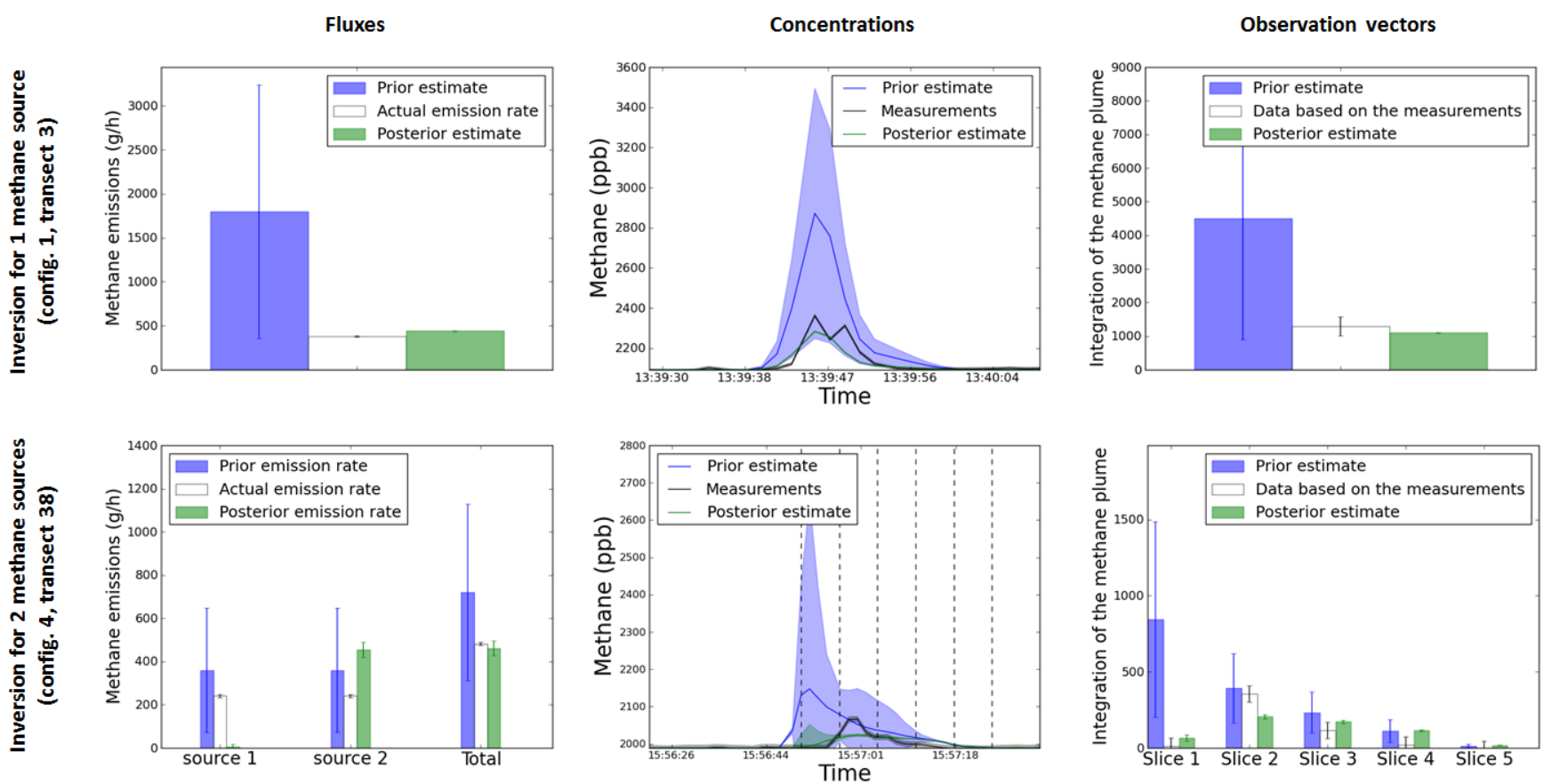

Figure 6. Examples of prior, posterior and measured values of emission rates, concentrations and values of the observation vector for cases in configuration 1 and 4 (observations from a single transect shown).

and tracer concentrations may also play a significant role in the level of error associated with the estimates from the tracer release technique. Instrument precision, on the other hand, should not significantly contribute to the error, since its amplitude is much smaller than the typical signals measured throughout the experiments (Figs. 1 and 5).

The relative differences between the actual rates and the tracer release estimates are much more important for the second and the fourth configurations at 32 and $67 \%$. The comparison between these results and those estimated in the first and third configurations indicates that in the latter cases, the observation operator errors associated with the mislocation of the tracer are much more important than the impact of the measurement and background errors. These error estimations based on direct comparison of the known emission rates are relatively well reflected by the uncertainty estimates, which are much lower for configurations 1 and 3 than for the second and fourth ones, both in terms of random error and in terms of biases.

\subsection{Combined approach}

\subsubsection{Configuration of the statistical inversion parameters}

In this section, we provide details on our definition of the prior estimate of the sources $\boldsymbol{f}^{b}$, of the covariance matrix of its uncertainties $\mathbf{B}$, and of the covariance matrix of the observation errors $\mathbf{R}$ that are needed for the application of Eq. (4) underlying the statistical inversion.
Here, we assume that the measurement and background errors are negligible compared to the transport errors, and thus that the observation errors can be represented solely by the transport errors. This assumption arose from the relatively high values taken by the transport error estimates. The modelled vs. measured tracer plumes indices and their product with the ratio between the measured methane and tracer plume indices are thus used to set up the variances of the observation error in the inversion configuration, i.e. the diagonal of the covariance matrix $\mathbf{R}$. In the case of a unique methane source, we use the absolute value of the difference between the modelled vs. measured plume indices to define the standard deviation of the observation error for the corresponding transect. When there are several methane sources within a site, we use the absolute value of the difference between the modelled vs. measured plume indices for each slice of the measurement transects (see Sect. 3.5). We assign a minimum value for these standard deviations to prevent one transect or slice of a transect dominating the others in the inversion process. In the least squares minimisation process associated with the statistical inversion, data assimilated with a considerably lower observation error than the others may fully drive the inversion results. For some transects, an excellent fit may occur between the model and the measurements in terms of plume indices (i.e. integration of the emission plume concentrations over the background) despite the shapes of the modelled and measured tracer plumes being significantly different, revealing some significant observation errors. Applying a threshold to the observation er- 
rors limits the impact of their underestimation through the objective comparison between the modelled and measured plume indices. We make the assumption that there is no correlation of the transport errors, and thus of the observation error (assuming that it is dominated by the transport errors) from one slice to the other slice of a given transect or from one transect to another one such that the $\mathbf{R}$ matrix is set up diagonally.

The typical prior knowledge $\boldsymbol{f}^{b}$ of the emission rate from waste treatment sites, farms, or gas extraction or compression sites from process models, typical national- to regional-scale factors is generally highly uncertain. It can bear more than $100 \%$ uncertainty and for many of these sites not even the order of magnitude is known. Despite working in the framework of a controlled release experiment, we attempt to set up the inversion system to have the same conditions as when monitoring the emissions from such sites. We thus set up the prior estimate of the methane emission rates to $1800 \mathrm{gh}^{-1}$ and the standard deviation of the prior uncertainty in these rates to $80 \%$ of this prior value. This ensures that the prior has a weak impact on the results. In general, there is no correlation between the prior uncertainties in the methane emissions from different targeted sources within a site, since they generally correspond to different processes (e.g. the aeration process and the clarification process in wastewater treatment plants; Yver Kwok et al., 2015). Therefore, here, the B matrix is set up diagonally.

\subsubsection{Results}

Figure 6 presents examples of results obtained using the combined statistical approach with one or several methane sources. The behaviour of the inversion system and the values in the concentration and observation space are illustrated for one transect only (for the third transect of the first configuration and for the 38th transect of the fourth configuration). It shows that the posterior estimates of the emissions have a much better fit of the simulated concentrations and plume indices than the prior emissions.

Table 2 presents the methane emission rates estimated with the combined approach for each tested configuration. We also analyse the covariance matrix $\mathbf{A}$ of the theoretical uncertainty in the emission estimates when using the statistical approach (Eq. 5), which provides a complementary assessment of the reliability of the results and of the level of separability between the two methane sources when using several of them in the experiments.

For the first and the third configurations, the statistical inversion gives similarly good estimates of the methane emission rates as the tracer release method, with relative deviations from the actual rates of 15 and $7 \%$. As expected, the tracer release technique provides better results for the first configuration. However the corresponding difference or relative error is very small and the combined statistical approach provides better results in the third configuration. Further- more, the combined approach derives relatively good estimates for the second and the fourth configurations as well, contrary to the tracer release method. Indeed, for these two experiments, the relative differences between the actual rates and the combined approach estimates are 16 and $4 \%$. Since being impacted by the background and measurement errors, this approach sill provides relative errors around $15 \%$ for configurations 1 and 2 but lower than $10 \%$ for the third and fourth configuration.

In all cases, the statistical inversion predicts a very low posterior uncertainty in the emission estimates for each configuration. For the fourth configuration with two methane sources, the approach fails to derive precise estimates of each source due to the significant overlapping of their emission plumes during most of the crossings. Indeed the system attributes almost all the emissions to one of the two sources and none to the other one. The diagnostic (through the computation of $\mathbf{A})$ of negative correlation $(-0.41)$ of the posterior uncertainties in these two sources supports the assumption that there is a weak ability to separate the signal from each source due to their overlapping, and that it is the main source of error in their individual estimates.

\section{Discussion}

The general results from these experiments indicate that both the tracer release technique and the combined statistical inversion system can provide good orders of magnitude of the total methane emission rates for each of the four source configurations that we have considered. However, when using the most favourable configurations of controlled emission where the methane source is collocated (configuration 1) or nearly aligned with the tracer source in the direction orthogonal to the wind direction (configurations 3 ) the results can still bear more than $10 \%$ relative errors. This is relatively high for the tracer release technique compared to what has been obtained, e.g. by Allen et al. (2013). For both the tracer release technique and the combined statistical inversion, the best results are not obtained for the most favourable controlled emission configuration when the acetylene and methane sources are collocated. This is surprising, since in such a configuration, the acetylene should provide a very precise (perfect if ignoring the measurement and background uncertainties) proxy of the atmospheric transport of methane. In the configurations with non-collocated sources, the results in the other configurations should be hampered by larger uncertainties in the representation of the atmospheric transport due to local variations in the wind between the methane and the acetylene sources.

The variations of the atmospheric conditions from one experimental configuration to the next reveal the strongest driver of the precision of the results in our study. It changes the turbulent patterns and thus the transport errors when using the model or when using the tracer with a mislocated source. It also changes the typical amplitude of the tracer 
and methane signals, and thus the signal-to-measurement and background noise ratio. This ratio is critical and strongly influences the inversion precisions, since for many measurement transects, our measurement and background errors appear to be significant compared to the amplitude of the measurements. The measurement precisions are a negligible source of error given the typical concentrations measured in this study. However, the small time lags between the acetylene and methane measurements are presumed to raise significant uncertainties in the comparison between acetylene and methane data. The variations in the background concentrations for methane but also in some cases for acetylene, also prove to be high enough to raise uncertainties in the single background value used for the computation of the so-called plume indices, i.e. the integral of the increase in the concentrations above the background within the plumes.

We anticipate that the results would have been better and more robust if the methane emission rates had been larger due to the increase in the signal-to-measurement and background noise ratio. In real application cases, the methane industrial emissions are definitely higher than the controlled emissions used in our experiments and we can thus expect the issue of the measurement and background errors to be less critical. Furthermore, we ignored these errors when deriving the covariance of the observation errors in the statistical inversions, although several indicators could have been used to characterise their statistics. We could thus help the combined statistical inversion system to better account for them when they are significant.

Despite these issues, this set of experiments clearly confirmed our expectations regarding the tracer release technique and the combined statistical inversion. In the configuration with the methane and acetylene sources collocated, the tracer release method provides better results than the statistical inversion since the latter is impacted by significant transport errors in addition to background and measurement errors while the tracer release technique is impacted by the last two sources of errors only. The optimisation of the Gaussian plume model using the acetylene data still proves to be efficient enough to limit the transport errors so that the accuracy of the statistical inversion is still close to that of the tracer release technique for the first experimental configuration.

In the other experimental configurations, which are representative of frequent situations in industrial sites when the tracer cannot be released close to the single or multiple targeted sources, the combined statistical inversion provides better results than the tracer release technique. Our OSSE demonstrates that the mislocation of the released tracer can induce large errors when considering moderate distances between the tracer and the targeted sources, even with much larger distances between the measurements and the sources. In these cases, our experiments with real data illustrated that the calibration of a Gaussian plume model using the tracer release method and the integration of the calibrated model in a statistical inversion framework help to reduce this error. The better behaviour of the statistical inversion compared to the tracer release technique cannot be explained by a stricter selection of the measurement transects by the former. We recomputed the results from the tracer release technique when limiting the selection of the transects to that of the combined statistical approach and found very similar results (33\% of error instead of $32 \%$ for the second configuration). On the contrary, the need to use a stricter selection of measurement transects that fit with the Gaussian plume model can be seen as a weakness of the combined inversion approach. The reduction of the transport error when using the model rather than the tracer with a mislocated source is the best explanation for the improvement of the results with the statistical inversion. These critical results demonstrate, in practice, the potential of our new method to provide better estimates than the traditional tracer release technique.

However, our results from the experiment with the fourth configuration of the controlled emissions fails to demonstrate the ability of the atmospheric inversion to provide precise estimates of the different emission rates from the multiple sources within our site. At least, it shows that the statistical inversion could diagnose by itself, with the estimate of the posterior uncertainty covariance matrix, indications that the two targeted sources of methane were too close, such that their plumes were hardly separated by the inversion in this fourth configuration.

The much lower uncertainties associated with the statistical inversion results seem to confirm that they are more robust than those from the tracer release method. However, even though the uncertainty estimates in both methods are supposed to cover all sources of uncertainties, they rely on very different assumptions regarding these sources of uncertainties and on very different theoretical derivations. In particular, the statistical inversion ignores biases, whereas we explicitly accounted for biases in the tracer release technique. Furthermore, unlike the estimate of uncertainties for the tracer release technique, the statistical inversion ignores the variations of methane model data misfits from one transect to the other, but these misfits could be an indicator of the uncertainties in the emissions. It strongly relies on our characterisation of the transport errors and prior uncertainties. We tried to rely on an objective quantification of the transport errors and we used such a high uncertainty in the prior flux estimates that this estimate did not have a large weight in the statistical inversion. However, the derivation of the transport error still relied on strong assumptions regarding its structure, and in particular regarding its spatio-temporal correlations. The computation of transport uncertainties using model-data misfits for tracer plume indices that are integrated over the whole plumes, i.e. for the same tracer plume indices as that used to optimise the transport model configuration, raises theoretical issues in the first three experimental configurations. It assumes that the dominant source of transport model errors is related to the inability of the transport model, in the 
range of parameterisation that are tested, to perfectly fit these plume indices. This does not account for the inability of the transport model to catch the turbulent patterns and the variations of the wind conditions in space and time. Assessing the transport model errors based on statistics of model-data misfits for slices of the tracer plume as in configuration 4 may help to better account for such sources of transport errors. However, in general, the information on the transport model errors from the tracer data may have to be complemented by other sources of information on the transport errors. This is in addition to information on the background and measurement uncertainties (as discussed above), which avoids underestimating the overall observation errors and thus the posterior uncertainties. All of this makes the comparison of the error bars for the two methods difficult and weakens the reliability on the quantification of the uncertainties in the results from the statistical inversions, especially since they appear to be very low for all experiments. These uncertainties should be used cautiously as an indicator of the relative behaviour of the system rather than as an absolute indicator of the result precision.

The promising results of this study should be seen as a proof of concept rather than as a comprehensive evaluation and assessment of its applicability in different situations. In particular, more complex situations with buildings and other obstacles disturbing the flow will require the application of more sophisticated transport models than the simple Gaussian plume model applied here, even though the overall concept of the combined statistical approach would still be valid.

Furthermore, as indicated above, the turbulent patterns induced significant transport errors that contributed to the uncertainties in the inversion results. The strict selection of the measurement transects that can be exploited by the inversion system is strongly related to the poor ability of the Gaussian plume model to simulate many of them. This is demanding in terms of measurements, with many transects needed to ensure that a significant set will be used for the statistical inversion.

At last, for the optimisation of the Gaussian plume model settings, the variable selection of stability classes representative of measurement transects shorter than $15 \mathrm{~min}$ is questionable. In the method, the fit of the model to the tracer data is the only critical criteria while the consistency between the stability class and the meteorological conditions according to the Pasquill table is just checked as a diagnostic that does not have any weight in the model optimisation. However, the changes in the resulting stability classes over short timescales make us question whether the Gaussian plume model is appropriate for such a combined inversion technique. A direct optimisation of the typical diffusion length of the Gaussian plume or of the parameters of the Briggs formulation, instead of the selection of the optimal stability class underlying such parameters, would allow a better, if not perfect, fit to the tracer plume indices. However, such an optimisation could increase the lack of physical consistency between the result- ing model parameters and the actual meteorological conditions due to the limitation of the Gaussian representation of the plumes.

All these problems contributed to the significant errors in the statistical inversions in this study and could make such errors too large in complex cases of actual industrial emission quantification. Therefore, while the choice of the Gaussian plume model for the initial tests to evaluate our new concept was appropriate, future studies should investigate how more complex models could be integrated in this inverse modelling framework. However, forcing CFD-driven dispersion models to fit the tracer data will not be straightforward, even if attempting to extract far more information from these data than simple plume indices. Even if modelling turbulent structures, the CFD models would be hardly controlled to fit that of the measurements. In general, the appropriate control techniques could be as complex as tracer data assimilation in these models which would make the method far more difficult to apply than in our study. This increase in complexity may make the method quite difficult to apply while there is a need for a precise and easy-to-implement methods for estimating methane emissions from the industrial sector. From this point of view, the tracer release technique definitely appears to be the most efficient technique.

Our concept faces another type of challenge. During measurement campaigns on actual industrial sites, the locations of the methane sources are not exactly known as in our tests. This lack of information could induce additional uncertainties to our estimates. Another source of uncertainty is the fact that in the tested configurations, methane point sources were used whereas during field campaigns, spread out and fugitive sources may be encountered while their spatial distribution could be poorly known. The lack of knowledge of the emission spatial distribution may decrease the advantage of the combined approach (which, in its present form strongly relies on this knowledge) compared to the release technique.

\section{Conclusions}

We propose a new atmospheric concentration measurementbased concept for instantaneous estimates of gas emissions from point sources or more generally from industrial sites. This concept combines the tracer release technique, localscale transport modelling and a statistical inversion framework. The idea is to optimise the model parameters based on the knowledge provided by the tracer release and concentration measurement and to exploit tracer model-measurement misfits to prescribe the statistics of the modelling error in the statistical inversion framework. Compared to the traditional tracer release technique, the method has the advantage of exploiting knowledge of the atmospheric transport provided by the known tracer release and measured concentration without relying on the collocation of the tracer emission and of the targeted gas emission. This is a critical advantage, since the tracer can hardly be collocated with the targeted sources 
in many of the real industrial cases. The statistical framework can account for the different sources of uncertainties in the source estimate, can solve different targeted sources together and can consider any valuable number of pieces of information in the measurement of the targeted gas for such an inversion.

We also propose a relatively simple implementation of this concept using a Gaussian plume model. Finally, we apply it to a series of controlled release experiments with methane and acetylene taken respectively as targeted and tracer gas and we compare its results to that of the tracer release technique to demonstrate the added benefit of our new approach. The results indicate that, when the tracer and targeted gas sources are collocated, the combined statistical approach yields results that are nearly as good as that from the tracer release technique, even though the former can be impacted by the transport modelling errors which do not apply to the latter. More importantly, the results confirm that, when the tracer and targeted gas sources are not collocated, the combined statistical approach provides better results than the tracer release technique. Such results with a rather simple implementation of the combined statistical approach using a Gaussian plume model are highly promising for our concept.

Our experiments fail to demonstrate the potential of this approach to estimate the different emission rates from the multiple sources within a site. Furthermore, as highlighted by Sect. 4, the robustness of the method and its assessment of the uncertainties needs to be improved. The generalisation of the method for applications to complex sites and meteorological conditions, based on more realistic transport models, will confront difficult technical and scientific challenges. However, at least our experiments promote further studies and development of our combined approach. They even promote application of our simple implementation framework to the instant quantification of real industrial sites emissions when the conditions are favourable for the use of a Gaussian plume model.

Data availability. Data are available on request from the authors.

Competing interests. The authors declare that they have no conflict of interest.

Acknowledgements. Funding for this research was provided by Climate-KIC. The authors thank Philippe Ciais and the industrial chair BridGES (Thales Alenia Space, Veolia, UVSQ, CEA, CNRS) for their support in this research. We would also like to acknowledge LSCE for the permission to conduct the measurements in the car park, particularly Pascal Doira for his availability and his help. Finally we thank the two anonymous reviewers, the associate editor Dominik Brunner, Levi Golston, Scott C. Herndon, Joseph R. Roscioli and Tara Yacovitch for their detailed and technical comments that helped to improve this study.
Edited by: Dominik Brunner

Reviewed by: two anonymous referees

\section{References}

Allen, D. T., Torres, V. M., Thomas, J., Sullivan, D. W., Harrison, M., Hendler, A., Herndon, S. C., Kolb, C. E., Fraser, M. P., Hill, A. D., Lamb, B. K., Miskimins, J., Sawyer, R. F., and Seinfeld, J. H.: Measurements of methane emissions at natural gas production sites in the United States, P. Natl. Acad. Sci. USA, 110, 17768-17773, https://doi.org/10.1073/pnas.1304880110, 2013.

Arya, S. P.: Air Pollution Meteorology and Dispersion, Oxford University Press, Oxford, UK, 1999.

Babilotte, A., Lagier, T., Fiani, E., and Taramini, V.: Fugitive Methane Emissions from Landfills: Field Comparison of Five Methods on a French Landfill, J. Environ. Eng., 136, 777-784, https://doi.org/10.1061/(ASCE)EE.1943-7870.0000260, 2010.

Baklanov, A. A. and Nuterman, R. B.: Multi-scale atmospheric environment modelling for urban areas, Adv. Sci. Res., 3, 53-57, https://doi.org/10.5194/asr-3-53-2009, 2009.

Bocquet, M.: An introduction to inverse modelling and parameter estimation for atmosphere and ocean sciences, vol. Special Issue, 461-493, https://doi.org/10.1093/acprof:oso/9780198723844.001.0001, 2012.

Bort, R. and Langeron, J.: Rapport National d'Inventaire pour la France au titre de la Convention cadre des Nations Unies sur les changements climatiques et du protocole de Kyoto, CITEPA, 2016.

Briggs, G. A.: Diffusion estimation of small emissions, Atmospheric Turbulence and Diffusion Laboratory Contribution, 83145, 1973.

Bréon, F. M., Broquet, G., Puygrenier, V., Chevallier, F., XuerefRemy, I., Ramonet, M., Dieudonné, E., Lopez, M., Schmidt, M., Perrussel, O., and Ciais, P.: An attempt at estimating Paris area $\mathrm{CO}_{2}$ emissions from atmospheric concentration measurements, Atmos. Chem. Phys., 15, 1707-1724, https://doi.org/10.5194/acp-15-1707-2015, 2015.

Chevallier, F., Bréon, F.-M., and Rayner, P. J.: Contribution of the Orbiting Carbon Observatory to the estimation of $\mathrm{CO}_{2}$ sources and sinks: Theoretical study in a variational data assimilation framework, J.Geophys. Res.-Atmos., 112, D09307, https://doi.org/10.1029/2006JD007375, 2007.

Czepiel, P. M., Mosher, B., Harriss, R. C., Shorter, J. H., McManus, J. B., Kolb, C. E., Allwine, E., and Lamb, B. K.: Landfill methane emissions measured by enclosure and atmospheric tracer methods, J. Geophys. Res.-Atmos., 101, 16711-16719, https://doi.org/10.1029/96JD00864, 1996.

Doury, A.: Une méthode de calcul pratique et générale pour la prévision des pollutions véhiculées par l'atmosphère, Rapport CEAR-4280, 1976.

Flesch, T. K., Wilson, J. D., Harper, L. A., Crenna, B. P., and Sharpe, R. R.: Deducing Ground-to-Air Emissions from Observed Trace Gas Concentrations: A Field Trial, J. Appl. Meteorol., 43, 487-502, https://doi.org/10.1175/15200450(2004)043<0487:DGEFOT>2.0.CO;2, 2004. 
Flesch, T. K., Wilson, J. D., Harper, L. A., Todd, R. W., and Cole, N. A.: Determining ammonia emissions from a cattle feedlot with an inverse dispersion technique, Agr. Forest Meteorol., 144, 139-155, https://doi.org/10.1016/j.agrformet.2007.02.006, 2007.

Gao, Z., Desjardins, R. L., and Flesch, T. K.: Comparison of a simplified micrometeorological mass difference technique and an inverse dispersion technique for estimating methane emissions from small area sources, Agr. Forest Meteorol., 149, 891-898, https://doi.org/10.1016/j.agrformet.2008.11.005, 2009.

Goetz, J. D., Floerchinger, C., Fortner, E. C., Wormhoudt, J., Massoli, P., Knighton, W. B., Herndon, S. C., Kolb, C. E., Knipping, E., Shaw, S. L., and DeCarlo, P. F.: Atmospheric Emission Characterization of Marcellus Shale Natural Gas Development Sites, Environ. Sci. Technol., 49, 7012-7020, https://doi.org/10.1021/acs.est.5b00452, 2015.

Goyal, A., Small, M. J., von Stackelberg, K., Burmistrov, D., and Jones, N.: Estimation of Fugitive Lead Emission Rates from Secondary Lead Facilities using Hierarchical Bayesian Models, Environ. Sci. Technol., 39, 4929-4937, https://doi.org/10.1021/es035465e, 2005.

Gurney, K. R., Law, R. M., Denning, A. S., Rayner, P. J., Baker, D., Bousquet, P., Bruhwiler, L., Chen, Y.-H., Ciais, P., Fan, S., Fung, I. Y., Gloor, M., Heimann, M., Higuchi, K., John, J., Kowalczyk, E., Maki, T., Maksyutov, S., Peylin, P., Prather, M., Pak, B. C., Sarmiento, J., Taguchi, S., Takahashi, T., and Yuen, C.-W.: TransCom $3 \mathrm{CO}_{2}$ inversion intercomparison: 1 . Annual mean control results and sensitivity to transport and prior flux information, Tellus B, 55, 555-579, https://doi.org/10.1034/j.16000889.2003.00049.x, 2003.

Hanna, S., White, J., Trolier, J., Vernot, R., Brown, M., Gowardhan, A., Kaplan, H., Alexander, Y., Moussafir, J., Wang, Y., Williamson, C., Hannan, J., and Hendrick, E.: Comparisons of JU2003 observations with four diagnostic urban wind flow and Lagrangian particle dispersion models, Atmos. Environ., 45, 4073-4081, https://doi.org/10.1016/j.atmosenv.2011.03.058, 2011.

IPCC: Climate Change 2013: The physical Science Basis. Contribution of Working Group I to the Fifth Assessment Report of the Intergovernmantal Panel on Climate Change, Cambridge University Press, Cambridge, UK and New York, NY, USA, 2013.

Korsakissok, I. and Mallet, V.: Comparative Study of Gaussian Dispersion Formulas within the Polyphemus Platform: Evaluation with Prairie Grass and Kincaid Experiments, J. Appl. Meteorol. Clim., 48, 2459-2473, https://doi.org/10.1175/2009JAMC2160.1, 2009.

Lamb, B., Westberg, H., and Allwine, G.: Isoprene emission fluxes determined by an atmospheric tracer technique, Atmos. Environ., 20, 1-8, https://doi.org/10.1016/0004-6981(86)90201-5, 1986.

Lamb, B. K., McManus, J. B., Shorter, J. H., Kolb, C. E., Mosher, B., Harriss, R. C., Allwine, E., Blaha, D., Howard, T., Guenther, A., Lott, R. A., Siverson, R., Westburg, H., and Zimmerman, P.: Development of Atmospheric Tracer Methods To Measure Methane Emissions from Natural Gas Facilities and Urban Areas, Environ. Sci. Technol., 29, 1468-1479, https://doi.org/10.1021/es00006a007, 1995.

Logan, J. A., Prather, M. J., Wofsy, S. C., and McElroy, M. B.: Tropospheric chemistry: A global per- spective, J. Geophys. Res.-Oceans, 86, 7210-7254, https://doi.org/10.1029/JC086iC08p07210, 1981.

Lushi, E. and Stockie, J. M.: An inverse Gaussian plume approach for estimating atmospheric pollutant emissions from multiple point sources, Atmos. Environ., 44, 1097-1107, https://doi.org/10.1016/j.atmosenv.2009.11.039, 2010.

Mallet, V., Quélo, D., Sportisse, B., Ahmed de Biasi, M., Debry, É., Korsakissok, I., Wu, L., Roustan, Y., Sartelet, K., Tombette, M., and Foudhil, H.: Technical Note: The air quality modeling system Polyphemus, Atmos. Chem. Phys., 7, 5479-5487, https://doi.org/10.5194/acp-7-5479-2007, 2007.

Marik, T. and Levin, I.: A new tracer experiment to estimate the methane emissions from a dairy cow shed using sulfur hexafluoride (SF6), Glob. Biogeochem. Cy., 10, 413-418, https://doi.org/10.1029/96GB01456, 1996.

Möllmann-Coers, M., Klemp, D., Mannschreck, K., and Slemr, F.: Determination of anthropogenic emissions in the Augsburg area by the source-tracer-ratio method, Atmospheric Environment, 36, Supplement 1, 95-107, https://doi.org/10.1016/S13522310(02)00212-1, 2002.

Mønster, J. G., Samuelsson, J., Kjeldsen, P., Rella, C. W., and Scheutz, C.: Quantifying methane emission from fugitive sources by combining tracer release and downwind measurements - A sensitivity analysis based on multiple field surveys, Waste Manage., 34, 1416-1428, https://doi.org/10.1016/j.wasman.2014.03.025, 2014.

Pasquill, F.: Estimation of the dispersion of windborne material, Meteorol. Mag., 90, 33-49, 1961.

Rayner, P. J., Enting, I. G., and Trudinger, C. M.: Optimizing the $\mathrm{CO}_{2}$ observing network for constraining sources and sinks, Tellus B, 48, 433-444, https://doi.org/10.1034/j.16000889.1996.t01-3-00003.x, 1996.

Roscioli, J. R., Yacovitch, T. I., Floerchinger, C., Mitchell, A. L., Tkacik, D. S., Subramanian, R., Martinez, D. M., Vaughn, T. L., Williams, L., Zimmerle, D., Robinson, A. L., Herndon, S. C., and Marchese, A. J.: Measurements of methane emissions from natural gas gathering facilities and processing plants: measurement methods, Atmos. Meas. Tech., 8, 20172035, https://doi.org/10.5194/amt-8-2017-2015, 2015.

Saunois, M., Bousquet, P., Poulter, B., Peregon, A., Ciais, P., Canadell, J. G., Dlugokencky, E. J., Etiope, G., Bastviken, D., Houweling, S., Janssens-Maenhout, G., Tubiello, F. N., Castaldi, S., Jackson, R. B., Alexe, M., Arora, V. K., Beerling, D. J., Bergamaschi, P., Blake, D. R., Brailsford, G., Brovkin, V., Bruhwiler, L., Crevoisier, C., Crill, P., Covey, K., Curry, C., Frankenberg, C., Gedney, N., Höglund-Isaksson, L., Ishizawa, M., Ito, A., Joos, F., Kim, H.-S., Kleinen, T., Krummel, P., Lamarque, J.-F., Langenfelds, R., Locatelli, R., Machida, T., Maksyutov, S., McDonald, K. C., Marshall, J., Melton, J. R., Morino, I., Naik, V., O’Doherty, S., Parmentier, F.-J. W., Patra, P. K., Peng, C., Peng, S., Peters, G. P., Pison, I., Prigent, C., Prinn, R., Ramonet, M., Riley, W. J., Saito, M., Santini, M., Schroeder, R., Simpson, I. J., Spahni, R., Steele, P., Takizawa, A., Thornton, B. F., Tian, H., Tohjima, Y., Viovy, N., Voulgarakis, A., van Weele, M., van der Werf, G. R., Weiss, R., Wiedinmyer, C., Wilton, D. J., Wiltshire, A., Worthy, D., Wunch, D., Xu, X., Yoshida, Y., Zhang, B., Zhang, Z., and Zhu, Q.: The global methane budget 2000-2012, Earth Syst. Sci. Data, 8, 697-751, https://doi.org/10.5194/essd-8-697-2016, 2016. 
Tarantola, A.: Inverse Problem Theory and Methods for Model Parameter Estimation, SIAM, google-Books-ID: qLSv6fpeMowC, 2005.

Whitby, R. A. and Altwicker, E. R.: Acetylene in the atmopshere: sources, representative ambient concentrations and ratios to other hydrocarbons, Atmos. Environ., 12, 1289-1296, 1977.
Yver Kwok, C. E., Müller, D., Caldow, C., Lebègue, B., Mønster, J. G., Rella, C. W., Scheutz, C., Schmidt, M., Ramonet, M., Warneke, T., Broquet, G., and Ciais, P.: Methane emission estimates using chamber and tracer release experiments for a municipal waste water treatment plant, Atmos. Meas. Tech., 8, 2853 2867, https://doi.org/10.5194/amt-8-2853-2015, 2015. 